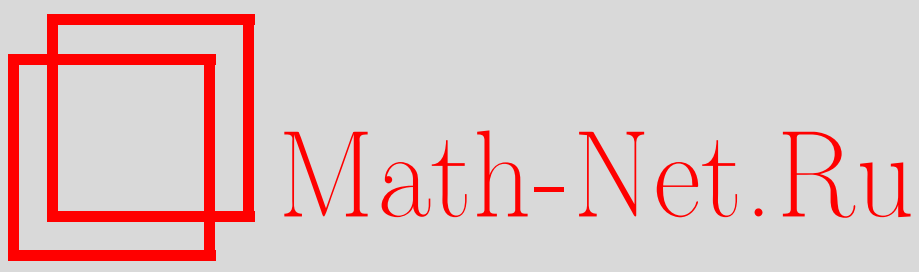

В. С. Климов, Ограниченные решения дифференциальных включений с однородной главной частью, Изв. РАН. Сер. матем., 2000, том 64, выпуск 4, 109-130

DOI: https://doi.org/10.4213/im296

Использование Общероссийского математического портала Math-Net.Ru подразумевает, что вы прочитали и согласны с пользовательским соглашением http://www . mathnet.ru/rus/agreement

Параметры загрузки:

IP : 54.224 .135 .184

26 апреля 2023 г., 18:23:29 
УДК 517.958

\author{
В. С. Климов
}

\title{
Ограниченные решения дифференциальных включений с однородной главной частью
}

\author{
Приводятся условия существования стационарных и ограниченных решений \\ дифференциальных включений с однородной главной частью. Устанавливаются \\ формулы для вычисления индекса нулевой особой точки однородного многознач- \\ ного векторного поля. \\ Библиографоя: 25 наименований.
}

В работе приводятся условия сушествования стационарных и ограниченных решений дифференциальных включений параболического типа с однородной главной частью. Устанавливаются формулы для вычисления индекса нулевой особой точки однородного многозначного векторного поля, порождаемого оператором монотонного типа [1]-[3]. Основу статьи составляют геометрические методы нелинейного анализа [4]-[7]. Близкие вопросы изучаются в [8], [9].

Используются обозначения: $\mathscr{B}(\mathfrak{R})$ - совокупность ограниченных подмножеств метрического пространства $\mathfrak{R} ; H_{l}(\mathfrak{R}), l=0,1, \ldots,-$ группы целочисленных гомологий с компактными носителями пространства $\mathfrak{R}$ (см. [10, гл. 9], [11, с. 316]); $b_{l}(\Re)$ - ранг групшы $H_{l}(\Re) ; \chi(\Re)=b_{0}(\Re)-b_{1}(\Re)+\cdots+(-1)^{l} b_{l}(\Re)+\cdots$ - эйлерова характеристика пространства $\mathfrak{R}$, имеюшая смысл лишь в случае $b_{l}(\mathfrak{R})<\infty \forall l$ и $b_{l}(\Re)=0$ при $l \geqslant l_{0} ; \overline{\mathscr{M}}(\stackrel{\circ}{\mathscr{M}}, \partial \mathscr{M})$ - замыкание (внутренность, гранища) множества $\mathscr{M} \subset \mathfrak{R} ; d_{\mathfrak{R}}(x, \mathscr{M})=\inf \{\rho(x, y), y \in \mathscr{M}\}-$ расстояние в метрике $\rho$ пространства $\mathfrak{R}$ от элемента $x$ до множества $\mathscr{M} ; \theta_{\mathfrak{R}}\left(\mathscr{M}_{1}, \mathscr{M}_{2}\right)=\sup \left\{d_{\mathfrak{R}}\left(x, \mathscr{M}_{2}\right), x \in \mathscr{M}_{1}\right\}-$ уклонение множества $\mathscr{M}_{1} \subset \mathfrak{R}$ от множества $\mathscr{M}_{2} \subset \mathfrak{R} ; \Gamma(X)$ - совокупность конечномерных подпространств банахова пространства $X ;\|x ; X\|$ - норма элемента $x$ в $X ; X^{*}$ - сопряженное к $X$ пространство; $\left(x, x^{*}\right)$ - значение функционала $x^{*} \in X^{*}$ на элементе $x \in X ; \sigma\left(X, X^{*}\right)$ и $\sigma\left(X^{*}, X\right)$ - слабые топологии на $X$ и $X^{*}$, порождаемые формой $(\cdot, \cdot)$; $\mathrm{Cv} X$ - совокупность выпуклых замкнутых подмножеств пространства $X ; s\left(x^{*}, \mathscr{M}\right)=\sup \left\{\left(x, x^{*}\right), x \in \mathscr{M}\right\}$ - опорная функция множества $\mathscr{M} \subset X ; L^{p}(T, X), 1 \leqslant p \leqslant \infty$, и $C(T, X)$ - банаховы пространства измеримых по Лебегу-Бохнеру (непрерывных) на отрезке $T \subset \mathbb{R}$ функций со значениями в пространстве $X$; как обычно, совпадаюшие п.в. (почти всюду) функции отождествляются, нормы в $L^{p}(T, X)$ и $C(T, X)$ вводятся стандартным образом (см., например, $[12$, с. 148,154$]) ; L_{w}^{p}(T, X), C_{w}(T, X)$ - топологические пространства слабо измеримых (слабо непрерывных) на отрезке $T \subset \mathbb{R}$ функций со значениями в $X$; определяющая в $L_{w}^{p}(T, X)\left(C_{w}(T, X)\right)$ топологию система полунорм задается равенством

$$
\|v\|_{l}=\left\|l(v) ; L^{p}(T)\right\| \quad\left(\|v\|_{l}=\|l(v) ; C(T)\|\right),
$$

где $l \in X^{*}, L^{p}(T)=L^{p}(T, \mathbb{R}), C(T)=C(T, \mathbb{R}) ;$ все банаховы пространства рассматриваются над полем $\mathbb{R}$ действительных чисел; $X_{1} \times X_{2}$ - прямое произведение $B$-пространств $X_{1}, X_{2}$; если $X_{1}, X_{2}$ вложены в топологическое простран- 
ство $X_{0}$, то с $X_{1}, X_{2}$ связаны $B$-пространства $X_{1} \cap X_{2}$ и $X_{1}+X_{2}[12$, с. 22,23$]$. Через $\Lambda(\mathscr{M}, Y)$ обозначается совокупность отображений множества $\mathscr{M} \subset X$ в банахово пространство $Y$, удовлетворяюших локальному условию Липшица, т.е. $F \in \Lambda(\mathscr{M}, Y)$, если для каждой точки $x$ из $\mathscr{M}$ найдутся такие ее окрестность $O$ и константа $L$, что

$$
\left\|F\left(x_{1}\right)-F\left(x_{2}\right)\right\| \leqslant L\left\|x_{1}-x_{2}\right\|, \quad x_{1} \in O, \quad x_{2} \in O .
$$

Класс $\Lambda(\mathscr{M}, \mathbb{R})$ действительных на $\mathscr{M}$ функций, удовлетворяющих локальному условию Липшица, обозначается символом $\Lambda(\mathscr{M})$.

Многозначное отображение $F$ множества $\mathscr{M}_{1}$ во множество $\mathscr{M}_{2}$ - это оператор, ставящий в соответствие элементу $x$ из $\mathscr{M}_{1}$ непустое множество $F(x) \subset \mathscr{M}_{2}$; если $\mathscr{M} \subset \mathscr{M}_{1}$, то $F(\mathscr{M})=\cup F(x) \quad(x \in \mathscr{M})$ - область значений отображения $F$ на множестве $\mathscr{M}$. Отображение $F: \mathscr{M}_{1} \rightarrow \mathscr{M}_{2}\left(\mathscr{M}_{i}\right.$ - подмножество метрического пространства $\left.\mathfrak{R}_{i}, \quad i=1,2\right)$ назьвают ограниченныц каждого $\mathscr{M}$ из $\mathscr{B}\left(\mathfrak{R}_{1}\right), \mathscr{M} \subset \mathscr{M}_{1}$. Через $I$ обозначается оператор тождественного преобразования.

1. Всюду далее $V$ - сепарабельное рефлексивное $B$-пространство, $\|\cdot\|$ и $\|\cdot\|_{*}-$ нормы в пространствах $V, V^{*}$ соответственно. Обозначим через $S(V)$ совокупность ограниченных многозначных операторов $\mathscr{F}: V \rightarrow \mathrm{Cv} V^{*}$, усиленно замкнутых в следующем смысле: для произвольных последовательностей $v_{n} \in V$, $v_{n}^{*} \in \mathscr{F}\left(v_{n}\right)$, обладающих свойствами

$$
v_{n} \rightarrow v \text { в } \sigma\left(V, V^{*}\right), \quad v_{n}^{*} \rightarrow v^{*} \text { в } \sigma\left(V^{*}, V\right), \quad \varlimsup_{n \rightarrow \infty}\left(v_{n}, v_{n}^{*}\right) \leqslant\left(v, v^{*}\right),
$$

имеют место соотношения: $v_{n} \rightarrow v$ в метрике $V, v^{*} \in \mathscr{F}(v)$.

Близкие классы однозначных операторов рассматривались в [1]-[3], [6], [12]. Примеры многозначных операторов класса $S(V)$ можно найти в [9], [13], [14]. Оператор $\mathscr{F}$ класса $S(V)$ хеминепрерывен на $V$ в следуюшем смысле: для любого $w$ из $V$ функция $s(w, \mathscr{F}(x))=\max \left\{\left(w, x^{*}\right), x^{*} \in \mathscr{F}(x)\right\}$ полунепрерывна сверху на $V$; в частности, график $\operatorname{Gr} \mathscr{F}=\left\{\left(x, x^{*}\right) \in V \times V^{*}\right\}$ оператора $\mathscr{F}$ замкнут в $V \times V^{*}$, где $V$ наделено сильной, а $V^{*}$ слабой топологиями [15, с. 128].

Элемент $v \in V$ назовем особой точкой оператора $\mathscr{F}$ класса $S(V)$, если $0 \in \mathscr{F}(v)$. Оператор $\mathscr{F}$ назовем невырожденным на множестве $\mathscr{M} \subset V$, если $\mathscr{M}$ не содержит особых точек. Множество $\mathscr{N}$, все точки которого являются особыми для $\mathscr{F}$, назовем особым. Пересечение множества особых точек поля $\mathscr{F}$ с шаром $\mathfrak{B}_{V}\left(x_{0}, R\right)=\left\{x \in V,\left\|x-x_{0}\right\| \leqslant R\right\}$ компактно [14].

ПРЕДЛОЖЕНИЕ 1 [9]. Пусть оператор $\mathscr{F}$ класса $S(V)$ невырожден на замкнутом множестве $\mathscr{M} \subset \stackrel{\circ}{\mathfrak{B}}_{V}\left(0, r_{0}\right)$. Тогда существуют $E$ из $\Gamma(V)$, отображение $w$ класса $\Lambda(\mathscr{M}, E)$ и постоянная $\delta>0$, обладающие свойствами

$$
\|w(x)\| \leqslant r_{0}, \quad s(w(x)-x, \mathscr{F}(x))<-\delta, \quad x \in \mathscr{M} .
$$

Пусть $\Omega$ - ограниченное открытое подмножество $V$ с гранищей $\partial \Omega$. Если оператор $\mathscr{F}$ класса $S(V)$ невырожден на $\partial \Omega$, то согласно предложению 1 существуют пространство $E$ класса $\Gamma(V)$ и векторное поле $w$ класса $\Lambda(\partial \Omega, E)$ такие, что

$$
\left(x-w(x), x^{*}\right)>0, \quad x \in \partial \Omega, \quad x^{*} \in \mathscr{F}(x) .
$$


В частности, поле $I-w$ невырождено на $\partial \Omega$ и определено вращение $\gamma(I-w, \partial \Omega)$ на $\partial \Omega[4],[5]$. Это вращение не зависит от произвола в способе выбора пространства $E$ и поля $w$ класса $\Lambda(\partial \Omega, E)$, удовлетворяюших условию острого угла (3). Число $\gamma(I-w, \partial \Omega)$ назовем вращением поля $\mathscr{F}$ на $\partial \Omega$ и обозначим символом $\gamma(\mathscr{F}, \Omega)$.

Последовательность $E_{n}, n=1,2, \ldots$, класса $\Gamma(V)$ назовем исчерпывающей пространство $V$, если $E_{n} \subset E_{n+1}, \quad n=1,2, \ldots$, и объединение всех пространств $E_{n}$ всюду плотно в пространстве $V$. Отметим, что в качестве пространства $E$, фигурируюшего в (2), (3), можно взять пространство $E_{n}$ при больших $n$ [9]. Несколько иные, но приводящие к тем же результатам, способы определения $\gamma(\mathscr{F}, \Omega)$ содержатся в [2], [3], [6], [14]. Врашение гомотопически инвариантно и аддитивно. Обсуждение этих и других свойств врашения можно найти в [2], [6], [9], [14]; здесь же приведем формулировки используемых далее результатов.

ПРЕДЛОЖЕНИЕ 2 . Пусть $\Omega$ - ограниченное открытое множество в пространстве $V$, оператор $\mathscr{F}$ класса $S(V)$ невырожден на $\partial \Omega$. Тогда:

1) если $\left(v-v_{0}, v^{*}\right) \geqslant 0 \quad\left(v \in \partial \Omega, v^{*} \in \mathscr{F}(v)\right)$ для некоторого $v_{0}$ из $\Omega$, то $\gamma(\mathscr{F}, \Omega)=1$ (свойство нормировки);

2) если область $\Omega$ симметрична относительно 0 , а поле $\mathscr{F}$ нечетно на $\partial \Omega(\mathscr{F}(-v)=-\mathscr{F}(v), v \in \partial \Omega)$, то $\gamma(\mathscr{F}, \Omega)$ - нечетное число (теорема об антиподах);

3) если $\gamma(\mathscr{F}, \Omega) \neq 0$, то включение $0 \in \mathscr{F}(v)$ имеет принадлежащее $\Omega$ решение (принцип ненулевого вращения);

4) если $\mathscr{F}_{0}, \mathscr{F}_{1} \in S(V)$ u $\theta_{V^{*}}\left(\mathscr{F}_{1}(v), \mathscr{F}_{0}(v)\right)<\theta_{V^{*}}\left(0, \mathscr{F}_{0}(v)\right), \quad v \in \partial \Omega$, mo $\gamma\left(\mathscr{F}_{1}, \Omega\right)=\gamma\left(\mathscr{F}_{0}, \Omega\right)$ (теорема Руше).

Если $\mathscr{N}$ - ограниченное изолированное особое множество поля $\mathscr{F}$ класса $S(V)$, то для ограниченных окрестностей $U$ множества $\mathscr{N}$ таких, что поле $\mathscr{F}$ невырождено на $U \backslash \mathscr{N}$, вращение $\gamma(\mathscr{F}, U)$ одинаково; оно называется индексом особого множества $\mathscr{N}$ поля $\mathscr{F}$ и обозначается символом ind $(\mathscr{N}, \mathscr{F})$. Если множество особых точек поля $\mathscr{F}$ ограничено, то вращение $\gamma\left(\mathscr{F}, \stackrel{\circ}{\mathfrak{B}}_{V}(0, R)\right)$ на сферах $\partial \mathfrak{B}_{V}(0, R)$ достаточно больших радиусов $R$ одинаково; оно обозначается символом ind $(\infty, \mathscr{F})$ и называется индексом на $\infty$ поля $\mathscr{F}$.

Вычисление индексов поля $\mathscr{F}$ в ряде случаев можно свести к аналогичной задаче для близкого к $\mathscr{F}$ однородного поля. Приведем некоторые определения. Оператор $\mathscr{A}: V \rightarrow \operatorname{Cv} V^{*}$ называется $m$-однороднымм $(m>0)$, если $\mathscr{A}(\lambda v)=\lambda^{m} \mathscr{A}(v)$ для любых $\lambda>0, v \in V$. Однородный оператор $\mathscr{A}$ регулярен на $\infty$, если включение $0 \in \mathscr{A}(v)$ имеет только нулевое решение. Регулярность $m$-однородного оператора $\mathscr{A}$ класса $S(V)$ влечет оценки

$$
k_{1}\|v\|^{m} \leqslant \theta_{V^{*}}(0, \mathscr{A}(v)), \quad \theta_{V^{*}}(\mathscr{A}(v), 0) \leqslant k_{2}\|v\|^{m}
$$

в которых $k_{1}, k_{2}$ - положительные константы.

Оператор $\mathscr{F}: V \rightarrow \mathrm{Cv} V^{*}$ называют асимптотически т-однородным, если найдется такой $m$-однородный оператор $\mathscr{A}: V \rightarrow \mathrm{Cv} V^{*}$, что

$$
\lim _{R \rightarrow \infty} \frac{1}{R^{m}} \sup _{\|v\| \leqslant R} \theta_{V^{*}}(\mathscr{F}(v), \mathscr{A}(v))=0
$$


оператор $\mathscr{A}$ называют главной частью оператора $\mathscr{F}$. Из (4), (5) вытекают неравенства

$$
c_{1}\|v\|^{m}-c_{0} \leqslant \theta_{V^{*}}(0, \mathscr{F}(v)), \quad \theta_{V^{*}}(\mathscr{F}(v), 0) \leqslant c_{2}\|v\|^{m}+c_{0}
$$

с некоторыми положительными константами $c_{i}$. Если $\mathscr{A}$ - регулярный $m$-однородный оператор класса $S(V)$, то 0 - единственная особая точка $\mathscr{A}$; в частности, $\operatorname{ind}(0, \mathscr{A})=\operatorname{ind}(\infty, \mathscr{A})$.

ТЕОРЕма 1. Пусть выполнены условия:

1) асимптотически т-однородный оператор $\mathscr{F}: V \rightarrow \mathrm{Cv} V^{*}$ и его главная часть $\mathscr{A}$ принадлежсат классу $S(V)$;

2) оператор $\mathscr{A}$ регулярен на $\infty u$ ind $(0, \mathscr{A}) \neq 0$.

Тогда оператор $\mathscr{F}$ есть сюргекиия $V$ на $V^{*}$, т.е. включение $v^{*} \in \mathscr{F}(v)$ разрешимо при любом $v^{*}$ из $V^{*}$.

ДоказАтельство. Из оценок (5), (6) и теоремы Руше следует, что на сферах $\partial \mathfrak{B}_{V}(0, R)$ больших радиусов $R$ векторные поля $\mathscr{F}(v)-v^{*}$ и $\mathscr{A}(v)$ имеют одинаковое врашение, поэтому ind $\left(\infty, \mathscr{F}(\cdot)-v^{*}\right)=\operatorname{ind}(\infty, \mathscr{A})=\operatorname{ind}(0, \mathscr{A}) \neq 0$. Теперь разрешимость включения $v^{*} \in \mathscr{F}(v)$ вытекает из принщипа ненулевого вращения. Теорема доказана.

СЛЕДСТВИЕ. Пусть выполнено условие 1) теоремы 1 и $\mathscr{A}$ - нечетный регулярный оператор. Тогда $\mathscr{F}(V)=V^{*}$.

Для однозначных операторов теорема 1 хорошо известна; достаточно полные ссылки можно найти в обзоре [16]. В условиях следствия ind $(0, \mathscr{A})$ - нечетное число, это вытекает из теоремы об антиподах. Рассмотрим более подробно задачу о вычислении индекса нулевой особой точки однородного оператора.

Пусть $\mathscr{A}, \mathscr{B}$ - регулярные на бесконечности однородные операторы класса $S(V)$ порядков однородности $m$ и $r$ соответственно, причем $m>r$. Если $x_{0}-$ ненулевая особая точка оператора $\mathscr{A}+\mathscr{B}$, то элемент $w_{0}=x_{0} /\left\|x_{0}\right\|$ есть нормированная особая точка оператора $\mathscr{A}+\lambda_{0} \mathscr{B}$, где $\lambda_{0}=\left\|x_{0}\right\|^{r-m}$. Верно и обратное: каждая нормированная особая точка $w_{0}$ оператора $\mathscr{A}+\lambda_{0} \mathscr{B}, \lambda_{0}>0$, порождает решение $x_{0}=\lambda_{0}^{1 /(r-m)} w_{0}$ включения $0 \in \mathscr{A}(v)+\mathscr{B}(v) ;$ при этом если $w_{0}-$ изолированная особая точка оператора $\mathscr{A}+\lambda_{0} \mathscr{B}$, то $x_{0}-$ изолированная особая точка поля $\mathscr{A}+\mathscr{B}$ и $\operatorname{ind}\left(w_{0}, \mathscr{A}+\lambda_{0} \mathscr{B}\right)=\operatorname{ind}\left(x_{0}, \mathscr{A}+\mathscr{B}\right)$.

Аналогичное замечание верно и в том случае, когда рассматривается изолированное особое множество $W_{0}$ поля $\mathscr{A}+\lambda_{0} \mathscr{B}$ :

$$
W_{0}=\left\{v \in V,\|v\|=1,0 \in \mathscr{A}(v)+\lambda_{0} \mathscr{B}(v)\right\} .
$$

Множество $X_{0}=\lambda_{0}^{1 /(r-m)} W_{0}$ есть изолированное особое множество поля $\mathscr{A}+\mathscr{B}$, при этом ind $\left(W_{0}, \mathscr{A}+\lambda_{0} \mathscr{B}\right)=\operatorname{ind}\left(X_{0}, \mathscr{A}+\mathscr{B}\right)$.

ТЕОРема 2. Пусть пара $\mathscr{A}, \mathscr{B}$ имеет конечное число нормированных собственных мнохеств $W_{1}, \ldots, W_{s}$, отвечающих положительным собственнымм значениям $\lambda_{1}, \ldots, \lambda_{s}$. Тогда

$$
\operatorname{ind}(0, \mathscr{A})=\operatorname{ind}(0, \mathscr{B})+\sum_{i=1}^{s} \operatorname{ind}\left(W_{i}, \mathscr{A}+\lambda_{i} \mathscr{B}\right)
$$


ДоказАТЕЛЬСтво. Так как $\mathscr{A}, \mathscr{B}$ - регулярные на $\infty$ операторы, то справедливы неравенства (4) и оценки $\theta_{V^{*}}(\mathscr{B}(v), 0) \leqslant k_{3}\|v\|^{r}, \theta_{V^{*}}(0, \mathscr{B}(v)) \geqslant k_{4}\|v\|^{r}$, $k_{3}, k_{4}$ - положительные константы. Из этих соотношений и теоремы Руше вытекают равенства

$$
\operatorname{ind}(0, \mathscr{A}+\mathscr{B})=\operatorname{ind}(0, \mathscr{B}), \quad \operatorname{ind}(\infty, \mathscr{A}+\mathscr{B})=\operatorname{ind}(\infty, \mathscr{A})
$$

Положим $X_{i}=\lambda_{i}^{1 /(r-m)} W_{i}, i=1, \ldots, s$. Тогда $X_{i}, i=1, \ldots, s,-$ изолированные ненулевые особые множества поля $\mathscr{A}+\mathscr{B}$. В силу теоремы об алгебраическом числе особых множеств [2]-[6] имеем

$$
\operatorname{ind}(\infty, \mathscr{A}+\mathscr{B})=\operatorname{ind}(0, \mathscr{A}+\mathscr{B})+\operatorname{ind}\left(X_{1}, \mathscr{A}+\mathscr{B}\right)+\cdots+\operatorname{ind}\left(X_{s}, \mathscr{A}+\mathscr{B}\right) .
$$

Теперь (7) следует из равенств

$$
\begin{gathered}
\operatorname{ind}(\infty, \mathscr{A}+\mathscr{B})=\operatorname{ind}(\infty, \mathscr{A})=\operatorname{ind}(0, \mathscr{A}) \\
\operatorname{ind}(0, \mathscr{A}+\mathscr{B})=\operatorname{ind}(0, \mathscr{B}), \quad \operatorname{ind}\left(X_{i}, \mathscr{A}+\mathscr{B}\right)=\operatorname{ind}\left(W_{i}, \mathscr{A}+\lambda_{i} \mathscr{B}\right) .
\end{gathered}
$$

Теорема доказана.

Аналогичный теореме 2 результат [5, с. 181] относится к случаю, когда $V$ конечномерное пространство, $\mathscr{A}$ - однозначный оператор, $\mathscr{B}$ - оператор тождественного преобразования. Если в условиях теоремы 2 оператор $\mathscr{B}$ монотонен, то $\left(v, v^{*}\right) \geqslant 0 \forall v^{*} \in \mathscr{B}(v)$, поэтому ind $(0, \mathscr{B})=1$ и $(7)$ приводит к равенству

$$
\operatorname{ind}(0, \mathscr{A})=1+\sum_{i=1}^{s} \operatorname{ind}\left(W_{i}, \mathscr{A}+\lambda_{i} \mathscr{B}\right)
$$

Аналогично, монотонность оператора $\mathscr{A}$ влечет равенство

$$
1=\operatorname{ind}(0, \mathscr{B})+\sum_{i=1}^{s} \operatorname{ind}\left(W_{i}, \mathscr{A}+\lambda_{i} \mathscr{B}\right) .
$$

Для вычисления индексов ind $\left(W_{i}, \mathscr{A}+\lambda_{i} \mathscr{B}\right)$ можно использовать известные методы [2]-[6]. Наиболее простым представляется случай, когда $W_{i}, i=1, \ldots, s,-$ одноточечные множества, $\mathscr{A}, \mathscr{B}$ - однозначные гладкие операторы.

2. Рассмотрим задачу о выгислении индекса потенциального векторного поля. Пусть $\Phi \in \Lambda(V), x \in V, v \in V$. Положим

$$
\Phi^{0}(x, v)=\varlimsup_{y \rightarrow x, t \rightarrow+0} \frac{\Phi(y+t v)-\Phi(y)}{t} .
$$

Число $\Phi^{0}(x, v)$ называют обобщенной производной функиионала $\Phi$ в точке $x$ по направлению $v$. Функционал $\Phi^{0}(x, \cdot): V \rightarrow \mathbb{R}$ есть опорная функция множества класса $\mathrm{Cv} V^{*}$, обозначаемого символом $\partial \Phi(x)$ и называемого обобщенным градиентом функционала $\Phi$ в точке $x[7$, с. 34].

Через $\Lambda_{1}(V)$ обозначим часть $\Lambda(V)$, состоящую из функционалов $\Phi$, для которых градиентное отображение $\mathscr{F}(x)=\partial \Phi(x), \quad x \in V$, принадлежит классу $S(V)$. В этом случае функционал $\Phi$ назовем потенциалом оператора $\mathscr{F}$, 
особые точки оператора $\mathscr{F}$ - критическими точками функиионала $\Phi$. Число с назовем критическим значением функиионала $\Phi$, если множество уровня $\{\Phi=c\}=\{v \in V, \Phi(v)=c\}$ содержит критическую точку $\Phi$, в противном случае $c$ назовем регулярным значением функиионала $\Phi$.

Пусть $b$ - регулярное значение функционала $\Phi$ класса $\Lambda_{1}(V)$ и нижнее лебегово множество $\mathscr{M}_{b}=\{x \in V, \Phi(x) \leqslant b\}$ ограничено. Тогда найдется такое число $a<b$, что отрезок $[a, b]$ не содержит критических значений функционала $\Phi$. В этом случае множество $\mathscr{M}_{a, b}=\{x \in V, a \leqslant \Phi(x) \leqslant b\}$ замкнуто и ограничено в пространстве $V$, а оператор $\mathscr{F}(x)=\partial \Phi(x)$ невырожден на $\mathscr{M}_{a, b}$. Согласно предложению 1 сушествуют пространство $E$ класса $\Gamma(V)$, векторное поле $w$ класса $\Lambda\left(\mathscr{M}_{a, b}, E\right)$ и постоянная $\delta>0$ такие, что $\Phi^{0}(x, w(x)-x)<-\delta$ и $\|w(x)\| \leqslant r_{0}$, где $r_{0}>\sup \left\{\|x\|, x \in \mathscr{M}_{a, b}\right\}$.

Из результатов работы [9] вытекает

ПРЕДЛОЖЕНИЕ 3. Пусть Г принадлежит $Г(V)$ и обладает указанными выше свойствами. Тогда:

1) пара $\left(\mathscr{M}_{a}, \mathscr{M}_{a} \cap E\right)$ есть деформационный ретракт пары $\left(\mathscr{M}_{b}, \mathscr{M}_{b} \cap E\right)$;

2) группь гомологий пространств $\mathscr{M}_{b}$ u $\mathscr{M}_{b} \cap E$ изоморфны: $H_{l}\left(\mathscr{M}_{b}\right) \approx$ $H_{l}\left(\mathscr{M}_{b} \cap E\right), \quad l=0,1, \ldots ;$ имеет смьсл әйлерова характеристика $\chi\left(\mathscr{M}_{b}\right)$ пространства $\mathscr{M}_{b}$ u $\gamma\left(\partial \Phi, \stackrel{\circ}{\mathscr{M}}_{b}\right)=\chi\left(\mathscr{M}_{b}\right)$.

Функционал $\Phi$ класса $\Lambda_{1}(V)$ назовем $p$-однородным $(p>1)$, если $\Phi(\lambda x)=$ $\lambda^{p} \Phi(x)$ для любых $\lambda>0, x \in V$; в этом случае $F=\partial \Phi$ есть $(p-1)$-однородный оператор класса $S(V)$. Справедливы равенства

$$
\Phi^{0}(v, v)=p \Phi(v)=-\Phi^{0}(v,-v)
$$

- естественные аналоги формулы Эйлера $\left(v, \Phi^{\prime}(v)\right)=p \Phi(v)$ для гладкого функционала $\Phi$. Очевидно, что 0 - критическая точка $p$-однородного функционала $\Phi$; если других критических точек нет, то функционал $\Phi$ назовем невырожсденым. Для невырожденного функционала $\Phi$ ind $(0, \partial \Phi)=\operatorname{ind}(\infty, \partial \Phi)$.

Если $\Phi(v) \geqslant 0 \forall v \in V$, то 0 - точка абсолютного минимума функционала $\Phi$, поэтому ind $(0, \partial \Phi)=1$ [14]. Рассмотрим случай, когда множество $\Re=\{v \in V$, $\|v\|=1, \Phi(v)<0\}$ непусто. Введем в $\Re$ метрику, индуцированную пространством $V$.

ТЕОРЕМА 3. Пусть $\Phi$ - невырожденный р-однородный функиионал класса $\Lambda_{1}(V)$ u $\Re \neq \varnothing$. Тогда:

1) существует такое пространство $E$ из $\Gamma(V)$, что $H_{l}(\Re) \approx H_{l}(\Re \cap E)$, $l=0,1, \ldots$;

2) имеет смысл әйлерова характеристика $\chi(\Re)$ пространства $\mathfrak{R} u$ $\operatorname{ind}(0, \partial \Phi)=1-\chi(\Re)$.

ДокАЗАТЕЛЬСТВо разобьем на несколько этапов.

1-й этап (вспомогательные функционалы). Не ограничивая обшности, можно считать, что $V, V^{*}$ - локально равномерно вьпуклые пространства. При выполнении этого предположения функционал $\Psi(x)=\|x\|^{2 p}$ непрерьвно дифференцируем на $V$, его производная $\Psi^{\prime}$ есть $(2 p-1)$-однородный регулярный на $\infty$ оператор класса $S(V)[14]$. Очевидно, что ind $\left(0, \Psi^{\prime}\right)=1$.

Введем в рассмотрение функционал $\Phi_{1}=\Phi+\Psi$. Поскольку $\partial \Phi_{1}=\partial \Phi+\Psi^{\prime}$, то $\Phi_{1} \in \Lambda_{1}(V), 0$ - изолированная критическая точка функционала $\Phi_{1}$ и $\operatorname{ind}\left(0, \partial \Phi_{1}\right)=$ 
$\operatorname{ind}(0, \partial \Phi)$. Множество $\mathscr{K}\left(\Phi_{1}\right)$ ненулевых критических точек $\Phi_{1}$ принадлежит внешности некоторого шара $\mathfrak{B}_{V}\left(0, \rho_{0}\right), \rho_{0}>0$, поэтому $\Psi(v) \geqslant \rho_{0}^{2 p}, v \in \mathscr{K}\left(\Phi_{1}\right)$. Если $v \in \mathscr{K}\left(\Phi_{1}\right)$, то $0 \in \partial \Phi(v)+\Psi^{\prime}(v)$, т.е. $-\Psi^{\prime}(v) \in \partial \Phi(v)$. В частности,

$$
2 p \Psi(v)=\left(-v,-\Psi^{\prime}(v)\right) \leqslant \Phi^{0}(v,-v)=-p \Phi(v),
$$

следовательно, $2 p \Psi(v)+p \Phi(v) \leqslant 0$. Отсюда получаем

$$
p \Phi_{1}(v) \leqslant-p \Psi(v) \leqslant-p \rho_{0}^{2 p}, \quad \Phi_{1}(v) \leqslant-\rho_{0}^{2 p} .
$$

Таким образом, $\mathscr{K}\left(\Phi_{1}\right) \subset\left\{v: \Phi_{1}(v) \leqslant-\rho_{0}^{2 p}\right\}$. Если $-\rho_{0}^{2 p}<b<0$, то $b-$ регулярное значение функционала $\Phi_{1} ; \mathscr{M}_{b}=\left\{v: \Phi_{1}(v) \leqslant b\right\} \subset \mathscr{M}_{0} \subset\{v:$ $\left.\|v\|<r_{0}\right\}$ и $\mathscr{K}\left(\Phi_{1}\right) \subset \stackrel{\circ}{\mathscr{M}}_{b}$.

2-й этап (построение пространства $E$ ). Согласно предложению 1 существуют $E_{0}$ из $\Gamma(V)$, отображение $w_{0}$ сферы $S_{1}=\{v \in V,\|v\|=1\}$ класса $\Lambda\left(S_{1}, E_{0}\right)$ и постоянная $\delta_{0}>0$, обладаюшие свойствами

$$
\left\|w_{0}(x)\right\| \leqslant 2, \quad \Phi^{0}\left(x, w_{0}(x)-x\right) \leqslant-\delta_{0} \quad \forall x \in S_{1} .
$$

Продолжим $w_{0}$ до 1-однородного отображения на все пространство $V$. За продолжением сохраним то же обозначение; таким образом, $w_{0} \in \Lambda\left(V, E_{0}\right),\left\|w_{0}(x)\right\| \leqslant$ $2\|x\|, \Phi^{0}\left(x, w_{0}(x)-x\right) \leqslant-\delta_{0}\|x\|^{p}$.

Фиксируем число $R>0$ такое, что $2 R<\rho_{0}$ и выполняется неравенство $3 r_{0}\left\|\Psi^{\prime}(x)\right\|_{*} \leqslant\left(\delta_{0} / 2\right)\|x\|^{p}$, если $\|x\| \leqslant 2 R$. Введем в рассмотрение множество $\mathscr{L}_{a}(R)=\left\{v \in V,\|v\| \geqslant R, a \leqslant \Phi_{1}(v) \leqslant 0\right\}$, где $-\rho_{0}^{2 p}<a<0$. Множество $\mathscr{L}_{a}(R)$ замкнуто и не содержит критических точек функционала $\Phi_{1} ;$ справедливы включения $\mathscr{L}_{a}(R) \subset \mathscr{M}_{0} \subset\left\{v:\|v\|<r_{0}\right\}$. В силу предложения 1 существуют $E_{1}$ из $\Gamma(V)$, отображение $w_{1}$ класса $\Lambda\left(\mathscr{L}_{a}(R), E_{1}\right)$ и постоянная $\delta_{1}>0$ такие, что $\left\|w_{1}(x)\right\| \leqslant r_{0}$ и $\Phi_{1}^{0}\left(x, w_{1}(x)-x\right)<-\delta_{1} \forall x \in \mathscr{L}_{a}(R)$.

Пусть $E=E_{0}+E_{1}$; очевидно, что $E \in \Gamma(V)$. Обозначим через $\lambda$ функцию на $[0, \infty)$, равную нулю на $[0, R]$ и единице на $[2 R, \infty)$ и линейную на отрезке $[R, 2 R]$. Положим

$$
w(x)=(1-\lambda(\|x\|)) w_{0}(x)+\lambda(\|x\|) w_{1}(x), \quad x \in \mathscr{M}_{0} ;
$$

так как $\lambda(s)=0$, если $0 \leqslant s \leqslant R$, способ продолжения отображения $w_{1}$ на шар $\|w\| \leqslant R$ несушествен. Справедливы соотношения

$$
\begin{aligned}
\Phi_{1}^{0}(x, w(x)-x) & \leqslant(1-\lambda(\|x\|)) \Phi_{1}^{0}\left(x, w_{0}(x)-x\right)+\lambda(\|x\|) \Phi_{1}^{0}\left(x, w_{1}(x)-x\right) \\
& \leqslant(1-\lambda(\|x\|))\left(\Psi^{\prime}\left(x, w_{0}(x)-x\right)+\Phi^{0}\left(x, w_{0}(x)-x\right)\right)-\delta_{1} \lambda(\|x\|) \\
& \leqslant(1-\lambda(\|x\|))\left(3 r_{0}\left\|\Psi^{\prime}(x)\right\|_{*}-\delta_{0}\|x\|^{p}\right)-\delta_{1} \lambda(\|x\|) \\
& \leqslant-(1-\lambda(\|x\|)) \frac{\delta_{0}}{2}\|x\|^{p}-\delta_{1} \lambda(\|x\|) \leqslant-\delta_{2}\|x\|^{p}
\end{aligned}
$$

с некоторой постоянной $\delta_{2}>0$. Отображение $w$ принадлежит классу $\Lambda\left(\mathscr{M}_{0}, E\right)$ и $\Phi_{1}^{0}(x, w(x)-x) \leqslant-\delta_{2}\|x\|^{p} ;$ в частности, $\sup \left\{\Phi_{1}^{0}(x, w(x)-x), a \leqslant \Phi_{1}(x) \leqslant b\right\}<0$ для любых $a, b$ из $\left(-\rho_{0}^{2 p}, 0\right)$.

3 -й этап (доказательство первого утверждения). Из предложения 3 следует, что пара $\left(\mathscr{M}_{a}, \mathscr{M}_{a} \cap E\right)$ есть деформационный ретракт пары $\left(\mathscr{M}_{b}, \mathscr{M}_{b} \cap E\right)$. Фиксируем 
целое число $l \geqslant 0$. Согласно предложению 3 группы $H_{l}\left(\mathscr{M}_{b}\right), H_{l}\left(\mathscr{M}_{b} \cap E\right), H_{l}\left(\mathscr{M}_{a}\right)$, $H_{l}\left(\mathscr{M}_{a} \cap E\right)$ изоморфны между собой. Порождаемый вложением $i_{a}^{b}:\left(\mathscr{M}_{a}, \mathscr{M}_{a} \cap\right.$ $E) \rightarrow\left(\mathscr{M}_{b}, \mathscr{M}_{b} \cap E\right)$ гомоморфизм $\left(i_{a}^{b}\right)^{*}: H_{l}\left(\mathscr{M}_{a}, \mathscr{M}_{a} \cap E\right) \rightarrow H_{l}\left(\mathscr{M}_{b}, \mathscr{M}_{b} \cap E\right)$ является изоморфизмом. Система $\left\{H_{l}\left(\mathscr{M}_{a}, \mathscr{M}_{a} \cap E\right),\left(i_{a}^{b}\right)^{*}\right\}$ образует прямой спектр на интервале $\left(-\rho_{0}^{2 p}, 0\right)$. Если $\Re_{1}=\left\{v: \Phi_{1}(v)<0\right\}$ - метрическое пространство с индуцированной $\|\cdot\|$ метрикой, то из определения групп гомологий с компактными носителями вытекает изоморфизм групп $H_{l}\left(\mathscr{M}_{b}\right), H_{l}\left(\mathscr{M}_{b} \cap E\right), H_{l}\left(\Re_{1}\right), l=0,1, \ldots$ В частности, из проведенных рассуждений вытекает, что имеют смысл и равны эйлеровы характеристики пространств $\mathscr{M}_{b}, \mathscr{M}_{b} \cap E, \Re_{1}$.

Покажем, что пространства $\mathfrak{R}$ и $\mathfrak{R}_{1}$ имеют одинаковый гомотопический тип. Если $v \in \mathfrak{R}_{1}$, то $\Phi(v)+\Psi(v)=\Phi_{1}(v)<0$, следовательно, $\Phi(v)<0$. Отображение $g_{1}(v)=v /\|v\|$ действует и непрерывно из $\mathfrak{R}_{1}$ в $\mathfrak{R}$. Для $v$ из $\mathfrak{R}$ положим

$$
T(v)=(-\Phi(v))^{\frac{1}{p}}, \quad g_{2}(v)=\frac{T(v)}{2} v .
$$

Отображение $g_{2}: \mathfrak{R} \rightarrow \mathfrak{R}_{1}$ непрерывно. Композиция $g_{1} \circ g_{2}: \mathfrak{R} \rightarrow \mathfrak{R}$ есть тождественное преобразование $\mathfrak{R}$, а композиция $g_{2} \circ g_{1}: \mathfrak{R}_{1} \rightarrow \mathfrak{R}_{1}$ гомотопно тождественному. Отсюда вытекает, что $\mathfrak{R}$ и $\mathfrak{R}_{1}$ имеют одинаковый гомотопический тип; то же самое верно и для пересечений $\Re \cap E, \mathfrak{R}_{1} \cap E$. Из проведенных рассуждений следует изоморфизм групп $H_{l}(\mathfrak{R}), H_{l}(\mathfrak{R} \cap E), H_{l}\left(\mathscr{M}_{b}\right), H_{l}\left(\mathscr{M}_{b} \cap E\right)$.

4-й этап (доказательство второго утверждения). Так как $H_{l}(\mathfrak{R}) \approx H_{l}(\Re \cap$ $E) \approx H_{l}\left(\mathscr{M}_{b}\right)$, то имеют смысл и совпадают эйлеровы характеристики соответствуюших пространств. Справедливы равенства (пояснения ниже)

$$
\begin{aligned}
1 & =\operatorname{ind}\left(\infty, \partial \Phi_{1}\right)=\operatorname{ind}\left(0, \partial \Phi_{1}\right)+\gamma\left(\partial \Phi_{1}, \stackrel{\circ}{\mathscr{M}}_{b}\right) \\
& =\operatorname{ind}(0, \partial \Phi)+\chi\left(\mathscr{M}_{b}\right)=\operatorname{ind}(0, \partial \Phi)+\chi(\Re) .
\end{aligned}
$$

Равенство $1=\operatorname{ind}\left(\infty, \partial \Phi_{1}\right)$ следует из того, что $\Phi_{1}-$ коэрцитивный функционал [9], [14]; равенство ind $\left(\infty, \partial \Phi_{1}\right)=\operatorname{ind}\left(0, \partial \Phi_{1}\right)+\gamma\left(\partial \Phi_{1}, \stackrel{\circ}{\mathscr{M}}_{b}\right)$ есть результат применения теоремы об алгебраическом числе особых точек к полю $\partial \Phi_{1}$; далее используются вытекаюшее из теоремы Руше равенство ind $\left(0, \partial \Phi_{1}\right)=\operatorname{ind}(0, \partial \Phi)$ и предложение 3 ; завершает доказательство отмечавшееся вьше равенство $\chi\left(\mathscr{M}_{b}\right)=$ $\chi(\Re)$. Итак, $1=\operatorname{ind}(0, \partial \Phi)+\chi(\Re)$. Теорема доказана.

Для гладкой однородной функции $\Phi$ конечного числа переменных формула $\operatorname{ind}\left(0, \Phi^{\prime}\right)=1-\chi(\Re)$ установлена в [8]. Если считать, что $\chi(\varnothing)=0$, то эта формула сохраняется и в случае $\mathfrak{R}=\varnothing$.

Если $E_{n}, n=1,2, \ldots,-$ исчерпьвающая $V$ последовательность класса $\Gamma(V)$, то в качестве пространства $E$, фигурируюшего в теореме 3 , можно взять пространство $E_{n}$ при $n>n_{0}$. Первое утверждение теоремы 3 влечет стабилизацию гомологических характеристик пространства $\mathfrak{R}$ при аппроксимации конечномерными сечениями.

Содержашиеся в теоремах 2,3 формулы вычисления индекса по обычным схемам [1]-[6] можно применить к исследованию включения $0 \in \mathscr{F}(x)$. Например, на их основе получаются оценки сверху и снизу числа решений этого включения, исследуется вопрос о сходимости метода Галёркина и т.п. 
3. Ниже $H$ - гильбертово пространство с нормой $|\cdot|$, пространство $V$ компактно и плотно вложено в $H$. Пространство $H$ далее отождествляется с ему сопряженным $H^{*}$, а $H^{*}=H-$ с некоторым подпространством сопряженного к $V$ пространства $V^{*}$. Число $\left(v, v^{*}\right)$ означает и скалярное произведение элементов $v$ и $v^{*}$ из $H$, и значение функционала $v^{*}$ из $V^{*}$ на элементе $v \in V$.

Пусть $T=[\alpha, \omega],-\infty<\alpha<\omega<\infty, 1<p<\infty, Y_{1}=L^{p}(T, V), Y_{2}=$ $L^{\infty}(T, H), \quad Z_{1}=L^{q}\left(T, V^{*}\right)(1 / p+1 / q=1), Z_{2}=L^{1}(T, H), \quad Y=Y_{1} \cap Y_{2}$, $Z=Z_{1}+Z_{2}$.

Пространство $Y$ ( $\left.Y_{1}\right)$ является сопряженным к пространству $Z\left(Z_{1}\right)$ [12]. Билинейная форма, определяюшая двойственность, задается равенством

$$
\langle y, z\rangle=\int_{T}(y(t), z(t)) d t .
$$

Если необходимо подчеркнуть зависимость пространств $Y, Z$ от $T$, будем обозначать их символами $Y(T)$ и $Z(T)$.

Положим $W=\left\{y \mid y \in Y, y^{\prime} \in Z\right\}$. Равенство $y^{\prime}+z=0$ означает, что $\left\langle y, \varphi^{\prime} v\right\rangle=\langle\varphi v, z\rangle, \varphi \in C_{0}^{\infty}(T), v \in V$. Класс $W$ вложен в $C(T, H)$; это позволяет ввести понятие значения функции из $W$ в каждой точке отрезка $T$. Если $y \in W$, то функция $t \rightarrow|y(t)|^{2}$ абсолютно непрерывна на $T$ и справедлива формула $\left(|y(t)|^{2}\right)^{\prime}=2\left(y(t), y^{\prime}(t)\right)$ п.в. [12], [13].

Пространство $Y$, наделенное слабейшей из топологий, мажорирующих топологии $\sigma(Y, Z)$ и $L_{w}^{p}(T, H)$, обозначается символом $Y_{0}$. Оно непрерывно вложено в $L^{p}(T, H)[17]$.

Введем некоторые классы многозначных операторов. Через $\alpha_{1}(Y, Z)$ обозначим совокупность операторов $F: Y \rightarrow \mathrm{Cv} Z$, удовлетворяющих условиям:

1) для каждого $\mathfrak{N}$ из $\mathscr{B}(Y)$ область значений $F(\mathfrak{N})=\cup F(y), y \in \mathfrak{N}$, предкомпактна в топологии $\sigma(Z, Y)$;

2) для произвольных последовательностей $y_{n} \in Y, z_{n} \in F\left(y_{n}\right)$, обладающих свойствами

$$
y_{n} \rightarrow y \text { в } Y_{0}, \quad z_{n} \rightarrow z \quad \text { в } \sigma(Z, Y), \quad \varlimsup_{n \rightarrow \infty}\left\langle y_{n}, z_{n}\right\rangle \leqslant\langle y, z\rangle,
$$

имеет место включение $z \in F(y)$ и сходимость $\left\langle 1_{[\alpha, t]} y_{n}, z_{n}\right\rangle \rightarrow\left\langle 1_{[\alpha, t]} y, z\right\rangle$ в $C(T)$ (здесь и далее $1_{\Delta}$ - характеристическая функция множества $\Delta \in \mathbb{R}$ ).

Условия 1), 2) выражают некоторые усиления свойств ограниченности и замкнутости оператора $F$. Условие усиленной ограниченности выполнено, если, например, оператор $F$ действует и ограничен из $Y$ в $Z_{1}+L^{r}(T, H), r>1$. Требование усиленной замкнутости оператора $F$ естественно для эллиптических операторов в дивергентной форме, рассматриваемых на пространствах Соболева (см., например, [1]-[3], [6], [12]).

Многозначный оператор $F: Y \rightarrow Z$ назовем локальныцм, если существует семейство операторов $\mathscr{F}(t): V \rightarrow V^{*}, t \in T$, порождающее $F$ в том смысле, что для любого $y$ из $Y$ множество $F(y)$ совпадает с множеством измеримых сечений отображения $t \rightarrow \mathscr{F}(t, y(t))$ (здесь и ниже $\mathscr{F}(t, v)=\mathscr{F}(t) v)$. Примеры локальных операторов класса $\alpha_{1}(Y, Z)$ приведены в [17], [18]; частный пример рассматривается в конце пункта.

Естественным образом определяется сужение $F_{I}$ локального оператора $F$ : $Y(T) \rightarrow Z(T)$ на пространство $Y(I)$, где $I \subset T$. Для любой функции $y$ из $Y(I)$ 
$F_{I}(y)$ есть множество измеримых сечений отображения $\mathscr{F}(t, y(t)), t \in I$, где $\mathscr{F}(t, \cdot)$ - порождаюшее оператор $F$ семейство отображений из $V$ в $V^{*}$. Как нетрудно видеть, если $F \in \alpha_{1}(Y, Z)$, то $F_{I}=\alpha_{1}(Y(I), Z(I))$.

Пусть $F$ - локальный оператор класса $\alpha_{1}(Y, Z)$, порождаемьй семейством отображений $\mathscr{F}(t, \cdot): V \rightarrow V^{*}, t \in T$. Рассмотрим дифференциальное включение

$$
0 \in y^{\prime}+\mathscr{F}(t, y)
$$

Под его решением будем понимать функцию $y$ класса $W$, для которой $-y^{\prime} \in F(y)$, или (что то же самое) $0 \in y^{\prime}+\mathscr{F}(t, y(t))$ п.в. Задача Коши для включения (9) заключается в отыскании решения $y$, удовлетворяющего условию

$$
y(\alpha)=h, \quad h \in H .
$$

Один из способов исследования задачи (9), (10) основан на построении ее аппроксимаций Галёркина и последующем предельном переходе. Пусть $E_{n}, n=$ $1,2, \ldots,-$ исчерпывающая $V$ последовательность конечномерных пространств, наделенных индуцированной из $H$ евклидовой структурой, $j_{n}: E_{n} \rightarrow V$ - оператор вложения, $j_{n}^{*}$ - сопряженный к нему оператор. Заменим включение (9) конечномерным дифференциальным включением

$$
0 \in x^{\prime}+j_{n}^{*} g_{n}
$$

под решением которого понимается функция $x_{n}$ класса $W$, удовлетворяюшая включению $x_{n}(t) \in E_{n}$ и равенству

$$
0=\left(v, x_{n}^{\prime}+g_{n}\right) \text { п.в. на } T \text {, }
$$

где $v$-произвольный элемент из $E_{n}$. Ниже

$$
d_{Y \times Z}\left(\left(x_{n} ; g_{n}\right) ; \operatorname{Gr} F\right)=\inf \left\{\left\|x_{n}-y ; Y\right\|+\left\|g_{n}-z ; Z\right\|, y \in Y, z \in F(y)\right\}
$$

- расстояние от $\left(x_{n} ; g_{n}\right) \in Y \times Z$ до графика отображения $F$.

Основополагающей для дальнейшего является

ЛЕмма 1. Пусть $x_{n}, \quad n=1,2, \ldots,-$ решение включения (11), $x_{n} \rightarrow x$ в $\sigma(Y, Z), d_{Y \times Z}\left(\left(x_{n} ; g_{n}\right) ; \operatorname{Gr} F\right) \rightarrow 0$ при $n \rightarrow \infty$. Тогда:

1) $x$ есть решение дифференциального включения (9) $u(t-\alpha) x_{n} \rightarrow(t-\alpha) x$ в $C(T, H)$;

2) если $x_{n}(\alpha) \rightarrow h$ в метрике $H$, то $x$ есть решение задачи Коши (9), (10) $u x_{n} \rightarrow x$ в $C(T, H)$.

ДокАЗАТЕЛЬСтво. Вначале установим справедливость второго утверждения леммы. Пусть $x_{n}(\alpha) \rightarrow h$ в метрике $H, z_{n} \in F\left(y_{n}\right)$ и $\left\|x_{n}-y_{n} ; Y\right\|+$ $\left\|g_{n}-z_{n} ; Z\right\| \rightarrow 0$ при $n \rightarrow \infty$. Из равенства (12) вытекает соотношение

$$
\left|x_{n}(t)\right|^{2}-\left|x_{n}(\alpha)\right|^{2}+2\left\langle 1_{[\alpha, t]} x_{n}, g_{n}\right\rangle=0
$$

и компактность последовательностей $x_{n}, y_{n}$ в пространстве $L_{w}^{p}(T, H)$. Без ограничения обшности можно считать, что

$$
\begin{gathered}
y_{n} \rightarrow x \quad \text { в } Y_{0}, \quad y_{n}(t) \rightarrow x(t) \quad \text { в } H \quad \text { п.в. на } T, \\
x_{n} \rightarrow x \quad \text { в } C_{w}(T, H), \quad z_{n} \rightarrow z \quad \text { в } \quad \sigma(Z, Y) .
\end{gathered}
$$


Используя (12), (14) и замкнутость оператора дифференширования, получаем, что $x$ принадлежит $W$ и удовлетворяет равенствам $x^{\prime}+z=0, x(\alpha)=h$, следствием которых является аналогичное (13) соотношение

$$
|x(t)|^{2}-|x(\alpha)|^{2}+2\left\langle 1_{[\alpha, t]} x, z\right\rangle=0
$$

Имеем, далее, оценки

$$
\varlimsup_{n \rightarrow \infty}\left\langle y_{n}, z_{n}\right\rangle=\varlimsup_{n \rightarrow \infty} \frac{\left|x_{n}(\alpha)\right|^{2}-\left|x_{n}(\omega)\right|^{2}}{2} \leqslant \frac{|x(\alpha)|^{2}-|x(\omega)|^{2}}{2}=\langle x, z\rangle,
$$

при выводе которых используется равенство (13) для $t=\omega$, сходимости $x_{n}(\alpha) \rightarrow$ $x(\alpha)$ в $H, x_{n}(\omega) \rightarrow x(\omega)$ в $\sigma(H, H)$ и равенство (15) при $t=\omega$. Последовательности $y_{n}, z_{n}$ обладают свойствами (8) (с заменой $y$ на $x$ ), поэтому справедливо включение $z \in F(x)$ и последовательность $\left\langle 1_{[\alpha, t]} y_{n}, z_{n}\right\rangle$ равномерно на $T$ сходится $\mathrm{K}\left\langle 1_{[\alpha, t]} x, z\right\rangle$. В соединении с (13)-(15) это влечет равномерную на отрезке $T$ сходимость $\left|x_{n}(t)\right|^{2} \rightarrow|x(t)|^{2}$. Поскольку $x_{n} \rightarrow x$ в $C_{w}(T, H)$, то $x_{n} \rightarrow x$ в $C(T, H)$. Второе утверждение леммы доказано.

Для доказательства первого утверждения леммы фиксируем отрезок $I=\left[t_{0}, \omega\right]$ так, что $\alpha<t_{0}$ и $x_{n}\left(t_{0}\right) \rightarrow x\left(t_{0}\right)$ в метрике $H$. Сужение $F_{I}$ оператора $F$ на пространство $Y(I)$ принадлежит $\alpha_{1}(Y(I), Z(I))$. Применяя уже доказанную часть леммы, получаем, что $x_{n} \rightarrow x$ в $C(I, H)$. Ввиду имеющегося произвола в способе выбора точки $t_{0}$, это влечет сходимость $(t-\alpha) x_{n} \rightarrow(t-\alpha) x$ в $C(T, H)$. Лемма доказана.

Включение (11) можно трактовать как приближение Галёркина к включению (9). Лемма 1 означает сходимость метода Галёркина при достаточно необременительных предположениях.

Рассмотрим многозначное отображение $\mathscr{F}: \mathbb{R} \times V \rightarrow \mathrm{Cv} V^{*}$, удовлетворяющее условиям:

I) $\mathscr{F}$ хеминепрерывно сверху;

II) справедлива оценка $\theta_{V^{*}}(\mathscr{F}(t, v), 0) \leqslant k\left(1+\|v\|^{p-1}\right)$, постоянные $k>0, p>1$ не зависят от $t, v$.

Из условия I) вытекает, что при любом $t \in \mathbb{R}$ отображение $\mathscr{F}(t, \cdot): V \rightarrow V^{*}$ хеминепрерывно, а при любом $v$ из $V$ отображение $\mathscr{F}(\cdot, v): \mathbb{R} \rightarrow V^{*}$ измеримо, в частности имеет измеримое сечение. Если $y: \mathbb{R} \rightarrow V$ - измеримая по Лебегу-Бохнеру функция, то множество $F(y)$ измеримых сечений многозначного отображения $t \rightarrow \mathscr{F}(t, y(t))$ непусто. Определяемый таким образом оператор суперпозиции $F$ локален; сохраним обозначение $F$ и для сужения этого оператора на пространство $L^{p}(T, V)$, где $T$ - произвольный отрезок действительной прямой. При выполнении условий I), II) $F: L^{p}(T, V) \rightarrow L^{q}\left(T, V^{*}\right)$ - ограниченный выпуклозначный оператор; его график Gr $F$ есть замкнутое подмножество $L^{p}(T, V) \times L^{q}\left(T, V^{*}\right)$, если наделить $L^{p}(T, V)$ сильной, а $L^{q}\left(T, V^{*}\right)$ слабой топологиями [15], [17].

Если не оговорено противное, всюду далее предполагается, что выполнены условия I), II). В случае конечномерного пространства $V$ оператор $F$ принадлежит классу $\alpha_{1}(Y(T), Z(T))$ для любого отрезка $T$. В бесконечномерном случае аналогичный факт верен при определенных дополнительных предположениях. Полезным оказывается следующий вариант условия эллиптичности отображения $\mathscr{F}(t, \cdot): V \rightarrow V^{*}[6],[19]:$ 
III) существуют такие константы $k_{0} \geqslant 0, k_{1}>0$, что

$$
\left(v_{1}-v_{2}, v_{1}^{*}-v_{2}^{*}\right)+k_{0}\left|v_{1}-v_{2}\right|^{p} \geqslant k_{1}\left\|v_{1}-v_{2}\right\|^{p},
$$

где $v_{i} \in V, v_{i}^{*} \in \mathscr{F}\left(t, v_{i}\right), i=1,2$. Оценка (16) есть нелинейньй аналог неравенства Гординга.

ЛЕмма 2. Пусть выполнены условия I)-III). Тогда:

1) $\mathscr{F}(t, \cdot) \in S(V)$ для каждого $t$;

2) порождаемыи $\mathscr{F}$ оператор суперпозичии $F: Y(t) \rightarrow Z(T)$ принадлежит классу $\alpha_{1}(Y(T), Z(T))$;

3) если $y_{n}, z_{n}$ обладают свойствами (8), то $y_{n} \rightarrow$ в в $L^{p}(T, V)$ для любого отрезка $T \subset \mathbb{R}$.

ДоКАЗАТЕЛЬСТво. Пусть $t \in \mathbb{R}, v_{n} \in V, v_{n}^{*} \in \mathscr{F}\left(t, v_{n}\right)$ - последовательности, обладаюшие свойствами (1). Фиксируем $v_{0}^{*}$ из $\mathscr{F}(t, v)$. Можно считать, что $v_{n} \rightarrow v$ в метрике $H$. В силу (16) имеем неравенство

$$
\left(v_{n}-v, v_{n}^{*}-v_{0}^{*}\right)+k_{0}\left|v_{n}-v\right|^{p} \geqslant k_{1}\left\|v_{n}-v\right\|^{p},
$$

левая часть которого в силу (1) стремится к нулю. Отсюда вытекает сходимость $v_{n} \rightarrow v$ в метрике пространства $V$. Поскольку оператор $\mathscr{F}(t, \cdot): V \rightarrow V^{*}$ хеминепрерывен сверху, то $v^{*} \in \mathscr{F}(t, v)$. Ограниченность оператора $\mathscr{F}(t, \cdot): V \rightarrow V^{*}$ следует из условия II).

Второе утверждение леммы вытекает из результатов работы [17]. Для доказательства третьего утверждения воспользуемся вытекающим из (16) неравенством

$$
\left(y_{n}(t)-y(t), z_{n}(t)-z(t)\right)+k_{0}\left|y_{n}(t)-y(t)\right|^{p} \geqslant k_{1}\left\|y_{n}(t)-y(t)\right\|^{p},
$$

где последовательности $y_{n}, z_{n}$ обладают свойствами (8), $z \in F(y)$. Интегрируя это равенство по отрезку $T$ и используя (8), получаем, что интегралы от левой части неравенства стремятся к нулю при $n \rightarrow \infty$. Отсюда следует сходимость $y_{n} \rightarrow y$ в $L^{p}(T, V)$. Лемма доказана.

СлЕДСТвИЕ. Если выполнены условия лемм 1, 2, то $x_{n} \rightarrow x$ в $L^{p}(T, V)$.

Более широкие, чем I)-III), условия, гарантируюшие включения $\mathscr{F}(t, \cdot) \in S(V)$, $F \in \alpha_{1}(Y, Z)$, можно найти в [2], [6], [17], [18].

4. В этом пункте $\mathscr{F}: \mathbb{R} \times V \rightarrow V^{*}$ - отображение, удовлетворяющее условиям I), II), порождаемый $\mathscr{F}$ оператор суперпозиции $F$ принадлежит классу $\alpha_{1}(Y(T), Z(T))$ для любого отрезка $T \subset \mathbb{R}$. Решение $y: \mathbb{R} \rightarrow V$ включения (9) назовем ограниченныц , если оно имеет конечную норму

$$
\left\|y ; C_{b}(\mathbb{R}, H)\right\|=\sup \{|y(t)|, t \in \mathbb{R}\} .
$$

Таким образом, если $y$ - ограниченное решение включения (9), то для любого отрезка $T \subset \mathbb{R}$ имеем $y \in W(T)$ и $\sup _{t}|y(t)|<\infty$.

Обозначим через $\mathfrak{M}_{p}(V)$ совокупность таких $(p-1)$-однородных отображений $\mathscr{A}: V \rightarrow \mathrm{Cv} V^{*}$, для которых не зависящее от $t$ отображение $\mathscr{F}(t, v)=\mathscr{A}(v)$ удовлетворяет условиям I)-III). Однородность оператора $\mathscr{A}$ влечет оценку $\theta_{V^{*}}(\mathscr{A}(v), 0) \leqslant k\|v\|^{p-1}$ с некоторой постоянной $k$. В силу леммы 2 порождаемый 
отображением $\mathscr{F}(t, v)=\mathscr{A}(v)$ оператор суперпозиции $A$ принадлежит классу $\alpha_{1}(Y(T), Z(T))$ для каждого отрезка $T$.

Если $\mathscr{A} \in \mathfrak{M}_{p}(V)$, то $0 \in \mathscr{A}(0)$, поэтому дифференциальное включение

$$
0 \in y^{\prime}+\mathscr{A}(y)
$$

имеет нулевое решение: $y(t) \equiv 0, t \in \mathbb{R}$. Обозначим через $\mathfrak{N}_{p}(V)$ часть класса $\mathfrak{M}_{p}(V)$, состоящую из таких операторов $\mathscr{A}$, для которых 0 - единственное ограниченное решение включения (17).

Для однозначных операторов в конечномерном пространстве аналогичный $\mathfrak{N}_{p}$ класс введен Н. Н. Красовским [20]. В последующем были установлены необходимые и достаточные условия принадлежности классу $\mathfrak{N}_{p}$ (см., например, [5] и приведенную там литературу). Примеры операторов данного класса в бесконечномерных пространствах рассматриваются в п. 6 .

Отображение $\mathscr{F}: \mathbb{R} \times V \rightarrow V^{*}$ назовем равномерно асимптотически $(p-1)$ -

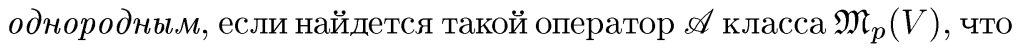

$$
\lim _{R \rightarrow \infty} \frac{1}{R^{p-1}} \sup _{\|v\| \leqslant R, t} \theta_{V^{*}}(\mathscr{F}(t, v), \mathscr{A}(v))=0
$$

В частности, $\mathscr{A}$ есть главная часть операторов $\mathscr{F}(t, \cdot): V \rightarrow V^{*}$. Равенство (18) эквивалентно сушествованию такой убывающей функции $K:(0,1] \rightarrow(0, \infty)$, что при любом $\varepsilon$ из $(0,1]$ верна оценка

$$
\theta_{V^{*}}(\mathscr{F}(t, v), \mathscr{A}(v)) \leqslant \varepsilon\|v\|^{p-1}+K(\varepsilon), \quad t \in \mathbb{R}, \quad v \in V .
$$

Далее наряду с отображением $\mathscr{F}$ окажется полезным рассматривать семейство отображений $\mathscr{F}_{R, \tau}, \quad R>0, \tau \in \mathbb{R}$, определяемое равенством $\mathscr{F}_{R, \tau}(t, v)=$ $R^{1-p} \mathscr{F}\left(\tau+R^{2-p} t, R v\right)$. Как нетрудно видеть, если $u(t)$ - решение включения (9), то функция $y(t)=(1 / R) u\left(\tau+R^{2-p} t\right)$ есть решение включения

$$
0 \in y^{\prime}+\mathscr{F}_{R, \tau}(t, y) \text {. }
$$

Исследуем зависимость от параметров $R, \tau$ операторов суперпозиции $F_{R, \tau}$, порождаемых отображениями $\mathscr{F} R, \tau: \mathbb{R} \times V \rightarrow V^{*}$. Введем пространство $\mathrm{BS}^{p}(\mathbb{R}, \mathscr{E})$ ограниченных по Степанову функций на $\mathbb{R}$ со значениями в $B$-пространстве $\mathscr{E}$. Оно состоит из измеримых по Лебегу-Бохнеру функций $f: \mathbb{R} \rightarrow \mathscr{E}$, для которых имеет смысл и конечна норма

$$
\left\|f ; \mathrm{BS}^{p}\right\|=\sup \left\{\left\|f ; L^{p}(I, \mathscr{E})\right\|\right\},
$$

где $\sup$ берется по всем отрезкам $I \subset \mathbb{R}$ единичной длины.

ЛЕмма 3. Пусть виполнено равенство $(18), \quad \mathfrak{B}=\left\{y \in \mathrm{BS}^{p}(\mathbb{R}, V)\right.$ : $\left.\left\|y ; \mathrm{BS}^{p}\right\| \leqslant 1\right\}$. Тогда

$$
\lim _{R \rightarrow \infty} \sup _{y \in \mathfrak{B}, \tau \in \mathbb{R}} \theta_{\mathrm{BS}^{q}\left(\mathbb{R}, V^{*}\right)}\left(F_{R, \tau}(y), A(y)\right)=0 .
$$


ДокАЗАТЕЛЬСтво. Фиксируем $\varepsilon>0$. Из (19) вытекает неравенство

$$
\theta_{V^{*}}(\mathscr{F}(t, R v), \mathscr{A}(R v)) \leqslant \varepsilon R^{p-1}\|v\|^{p-1}+K(\varepsilon),
$$

верное для любого $R>0$. Если $R>R_{1}(\varepsilon)=\sqrt[p-1]{K(\varepsilon) / \varepsilon}$, то приходим к неравенству

$$
\theta_{V^{*}}\left(\mathscr{F}_{R, \tau}(t, v), \mathscr{A}(v)\right)<\varepsilon\left(1+\|v\|^{p-1}\right) .
$$

Пусть $y \in \mathfrak{B}, R>R_{1}(\varepsilon), \tau \in \mathbb{R}, f(t)$ - измеримое сечение многозначного отображения $\mathscr{F} R, \tau(t, y(t))$. Из оценки $(22)$ следует соотношение

$$
\theta_{V^{*}}(f(t), \mathscr{A}(y(t)))<\varepsilon\left(1+\|y(t)\|^{p-1}\right) .
$$

Применяя (23) и известные результаты об измеримом выборе [21], найдем такое измеримое сечение $z(t)$ многозначного отображения $t \rightarrow \mathscr{A}(y(t))$, что $\| f(t)-$ $z(t) \|<\varepsilon\left(1+\|y(t)\|^{p-1}\right)$. Это неравенство и условие $\| y$; $\mathrm{BS}^{p} \| \leqslant 1$ влекут оценки

$$
\left\|f-z ; \mathrm{BS}^{q}\right\|<2 \varepsilon, \quad \theta_{\mathrm{BS}^{q}\left(\mathbb{R}, V^{*}\right)}\left(F_{R, \tau}(y), A(y)\right) \leqslant 2 \varepsilon
$$

при $R>R_{1}(\varepsilon)$. Лемма доказана.

Из равенства (18) и включения $\mathscr{A} \in \mathfrak{M}_{p}(V)$ вытекает оценка

$$
\left(v, v^{*}\right)+\varkappa_{0}|v|^{p} \geqslant \varkappa_{1}\|v\|^{p}-\varkappa_{2},
$$

в которой $v \in V, v^{*} \in \mathscr{F}_{R, \tau}(t, v), \varkappa_{i}$ - положительные константы, не зависяшие от $t, v, \tau, R$. Если $y$ - ограниченное решение включения (20), то $y^{\prime}=-z$, где $z(t) \in \mathscr{F} R, \tau(t, y(t))$ п.в. В этом случае

$$
\frac{1}{2}\left(|y(t)|^{2}\right)^{\prime}=-(y(t), z(t)) \leqslant \varkappa_{0}|y(t)|^{p}-\varkappa_{1}\|y(t)\|^{p}-\varkappa_{2}
$$

и, следовательно,

$$
\varkappa_{1} \int_{t_{0}}^{t_{1}}\|y(t)\|^{p} d t \leqslant \varkappa_{0} \int_{t_{0}}^{t_{1}}|y(t)|^{p} d t-\varkappa_{2}\left(t_{1}-t_{0}\right)-\frac{\left|y\left(t_{1}\right)\right|^{2}-\left|y\left(t_{0}\right)\right|^{2}}{2} .
$$

Последнее неравенство влечет включение $y \in \mathrm{BS}^{p}(\mathbb{R}, V)$ и оценку $\left\|y ; \mathrm{BS}^{p}\right\| \leqslant$ $\psi\left(\left\|y ; C_{b}(\mathbb{R}, H)\right\|\right)$, где $\psi: \mathbb{R}_{+} \rightarrow \mathbb{R}_{+}$- возрастаюшая функция, зависяшая лишь от фигурируюших в $(24)$ постоянных $\varkappa_{0}, \varkappa_{1}, \varkappa_{2}$. Таким образом, ограниченное решение включения (20) автоматически оказьвается и ограниченным по Степанову; функция $\psi$ характеризует связь норм решений $y$ в пространствах $C_{b}(\mathbb{R}, H)$ и $\mathrm{BS}^{p}(\mathbb{R}, V)$.

Существование ограниченных решений дифференциального включения (9) может быть доказано с помошью метода Галёркина. Пусть $E_{n}$ - исчерпьвающая $V$ последовательность конечномерных пространств, наделенных индуцированной из $H$ евклидовой структурой, $j_{n}: E_{n} \rightarrow V$ - оператор вложения, $j_{n}^{*}: V^{*} \rightarrow E_{n}-$ сопряженный к нему оператор. Рассматриваются аппроксимации Галёркина вида

$$
0 \in u^{\prime}+j_{n}^{*} F\left(j_{n} u\right)
$$

Под ограниченным решением включения (25) понимается абсолютно непрерывная и ограниченная на $\mathbb{R}$ функция $u: \mathbb{R} \rightarrow E_{n}$, для которой $0 \in$ $u^{\prime}(t)+j_{n}^{*} \mathscr{F}(t, u(t))$ п.в. на $\mathbb{R}$. Для подобных решений справедлива оценка $\left\|u ; \mathrm{BS}^{p}\right\| \leqslant \psi\left(\left\|u ; C_{b}(\mathbb{R}, H)\right\|\right)$; предшествуюшие рассуждения сохраняют силу. Важно заметить, что функция $\psi$ не зависит от $n$. 
Лемма 4. Пусть выполнено неравенство (24) и $\mathscr{A} \in \mathfrak{N}_{p}(V)$. Пусть для каждого $n$ включение (25) имеет ограниченное решение $u_{n}$. Тогда и включение (9) имеет ограниченное решение.

ДоКАЗАТЕЛЬСТВО. Положим $R_{n}=\left\|u_{n} ; C_{b}(\mathbb{R}, H)\right\|$ и установим ограниченность последовательности $R_{n}$. В предположении противного можно без ограничения обшности считать, что $R_{n} \rightarrow \infty$ и $R_{n}>1$. Фиксируем $\tau_{n} \in \mathbb{R}$ таким, что $\left|u_{n}\left(\tau_{n}\right)\right|>R_{n} / 2$. Положим

$$
y_{n}(t)=\frac{u_{n}\left(\tau_{n}+R_{n}^{2-p} t\right)}{R_{n}}, \quad n=1,2, \ldots, \quad t \in \mathbb{R} .
$$

Очевидно, $\left\|y_{n} ; C_{b}(\mathbb{R}, H)\right\|=1,\left|y_{n}(0)\right|>1 / 2$ и $y_{n}$ - решение дифференциального включения

$$
0 \in y_{n}^{\prime}+j_{n}^{*} F_{R_{n}, \tau_{n}}\left(j_{n} y_{n}\right) \text {. }
$$

Из (26) вытекает оценка $\left\|y_{n} ; \mathrm{BS}^{p}\right\| \leqslant \psi(1)$ и равенство $0=y_{n}^{\prime}+j_{n}^{*} f_{n}$, где $f_{n} \in F_{R_{n}, \tau_{n}}\left(j_{n} y_{n}\right)$.

В силу леммы $3 \theta_{\mathrm{BS}^{q}}\left(f_{n}, A\left(y_{n}\right)\right) \rightarrow 0$; включение $0 \in y^{\prime}+j_{n}^{*} f_{n}$ аналогично включению (11) и возникает при замене $F$ на $A$. Не ограничивая общности, можно считать, что для любого отрезка $T=[\alpha, \omega] \subset \mathbb{R}$ выполнены соотношения $y_{n} \rightarrow y$ в $\sigma(Y(T), Z(T)), z_{n} \rightarrow z$ в $\sigma(Z(T), Y(T))$. Из леммы 1 следует тогда, что предельная функция $y$ есть решение дифференциального включения $(17)$ и $(t-\alpha) y_{n}(t) \rightarrow(t-\alpha) y(t)$ в $C(T, H)$. Полагая $\alpha=-1, \omega=1$, получаем, что $y_{n}(0) \rightarrow y(0)$ в $H$, поэтому $|y(0)| \geqslant 1 / 2$, т.е. $y$ - ненулевое решение включения (17). Это противоречит условию $\mathscr{A} \in \mathfrak{N}_{p}(V)$. Полученное противоречие доказывает ограниченность последовательности $u_{n}$ в пространстве $C_{b}(\mathbb{R}, H):\left\|u_{n} ; C_{b}(\mathbb{R}, H)\right\| \leqslant R_{0}$. Последовательность $u_{n}$ ограничена и в пространстве $\mathrm{BS}^{p}(R, V):\left\|u_{n} ; \mathrm{BS}^{p}\right\| \leqslant \psi\left(R_{0}\right)$.

Можно считать, что $u_{n} \rightarrow u$ в $\sigma(Y(T), Z(T))$ для любого отрезка $T, u_{n}^{\prime}=$ $-j_{n}^{*} g_{n}, g_{n} \in F\left(u_{n}\right)$ и $g_{n} \rightarrow g$ в $\sigma(Z(T), Y(T))$. Снова применяя лемму 1 , получаем, что $u$ - решение включения (9) и $\left\|u ; C_{b}(\mathbb{R}, H)\right\| \leqslant R_{0}$. Лемма доказана.

Лемма 5. Если $\mathscr{A} \in \mathfrak{N}_{p}(V)$, то $j_{n}^{*} \mathscr{A} j_{n} \in \mathfrak{N}_{p}\left(E_{n}\right)$ при достаточно больuux $n$.

ДоКАЗАТЕЛЬСТВО. В предположении противного для бесконечного числа индексов $n$ включение $0 \in u^{\prime}+j_{n}^{*} \mathscr{A}\left(j_{n} u\right)$ имеет ненулевое ограниченное решение $u_{n}$. Будем считать, что это верно при любом $n$, и положим $R_{n}=\left\|u_{n} ; C_{b}(\mathbb{R}, H)\right\|$. Подберем $\tau_{n}$ так, что $\left|u_{n}\left(\tau_{n}\right)\right|>R_{n} / 2$. Вектор-функция $y_{n}(t)=\left(1 / R_{n}\right) u_{n}\left(\tau_{n}+\right.$ $\left.R_{n}^{2-p} t\right)$ удовлетворяет соотношениям $0 \in y_{n}^{\prime}+j_{n}^{*} \mathscr{A}\left(j_{n} y_{n}\right), \quad\left|y_{n}(0)\right|>1 / 2$, $\left|y_{n}(t)\right| \leqslant 1$. Отсюда вытекает ограниченность последовательности $y_{n}$ в пространствах $C_{b}(\mathbb{R}, H), \mathrm{BS}^{p}(\mathbb{R}, V)$. Без ограничения обшности можно считать, что $y_{n} \rightarrow y$ в $\sigma(Y(T), Z(T))$ для любого отрезка $T \subset \mathbb{R}$. В силу леммы $1 y$-ограниченное решение дифференщиального включения $(17)$, при этом $y_{n}(0) \rightarrow y(0)$ в метрике $H$, следовательно, $|y(0)| \geqslant 1 / 2$. Таким образом, $y$ есть ненулевое ограниченное решение включения (17), а это противоречит условию $\mathscr{A} \in \mathfrak{N}_{p}(V)$. Лемма доказана. 
5. Пусть $E$ - конечномерное евклидово пространство, $(\cdot, \cdot)$ и $|\cdot|$ - скалярное произведение и евклидова норма в пространстве $E, E^{2}=E \times E$-прямое произведение евклидова пространства $E$ на себя, $\theta_{E^{2}}\left(\mathscr{M}_{1}, \mathscr{M}_{2}\right)$ - уклонение множества $\mathscr{M}_{1}$ от множества $\mathscr{M}_{2}$ в метрике пространства $E^{2}$. Изучается дифференциальное включение $(9)$, где $\mathscr{F}: \mathbb{R} \times E \rightarrow \mathrm{Cv} E$ - многозначное отображение, удовлетворяющее условиям I), II) (с естественной заменой пространств $V, H, V^{*}$ пространством $E$; аналогичное замечание относится и к последуюшим конструкциям).

Как нетрудно видеть, оператор $\mathscr{A}: E \rightarrow \mathrm{Kv} E$ принадлежит классу $\mathfrak{M}_{p}(E)$, если и только если он полунепрерывен сверху и $(p-1)$-однороден. Через $\theta\left(\mathscr{A}_{1}, \mathscr{A}_{2}\right)$, $\mathscr{A}_{i} \in \mathfrak{M}_{p}(E)$, будем обозначать уклонение $\theta_{E^{2}}\left(\operatorname{Gr} \mathscr{A}_{1}, \operatorname{Gr} \mathscr{A}_{2}\right)$ графика $\operatorname{Gr} \mathscr{A}_{1}$ оператора $\mathscr{A}_{1}$ на шаре $\{v:|v| \leqslant 1\}$ от графика $\operatorname{Gr} \mathscr{A}_{2}$ оператора $\mathscr{A}_{2}$ на том же шаре. Отметим некоторые свойства уклонения. Справедлив вариант неравенства треугольника:

$$
\theta\left(\mathscr{A}_{1}, \mathscr{A}_{2}\right) \leqslant \theta\left(\mathscr{A}_{1}, \mathscr{A}_{3}\right)+\theta\left(\mathscr{A}_{3}, \mathscr{A}_{2}\right), \quad \mathscr{A}_{i} \in \mathfrak{M}_{p}(E), \quad i=1,2,3
$$

Множество однозначных операторов плотно в $\mathfrak{M}_{p}(E)$ : для каждого оператора $\mathscr{A}$ из $\mathfrak{M}_{p}(E)$ и каждого $\delta>0$ сушествует такой однозначный оператор $\mathscr{A}^{\delta}$ из $\mathfrak{M}_{p}(E)$, что $\theta\left(\mathscr{A}^{\delta}, \mathscr{A}\right)<\delta$. Если $\theta\left(\mathscr{A}_{i}, \mathscr{A}\right) \rightarrow 0$ при $i \rightarrow \infty$ и $\mathscr{B}_{i}=\operatorname{co}\left(\mathscr{A}_{i}, \mathscr{A}\right)\left(\mathscr{B}_{i}(v)=\right.$ $\left.\operatorname{co}\left(\mathscr{A}_{i}(v) \cup \mathscr{A}(v)\right)\right)$, то $\theta\left(\mathscr{B}_{i}, \mathscr{A}\right) \rightarrow 0$. Как и в п. $4, \mathfrak{N}_{p}(E)$ - часть $\mathfrak{M}_{p}(E)$, состояшая из таких операторов $\mathscr{A}$, что дифференциальное включение (17) не имеет ненулевых ограниченных решений. Класс $\mathfrak{N}_{p}(E)$ устойчив по отношению к малым уклонениям. Более точно, справедлива

Лемма 6. Пусть $\mathscr{A}_{0} \in \mathfrak{N}_{p}(E)$. Тогда найдется такое $\delta=\delta\left(\mathscr{A}_{0}\right)>0$, что если $\theta\left(\mathscr{A}, \mathscr{A}_{0}\right)<\delta$, mo $\mathscr{A} \in \mathfrak{N}_{p}(E)$.

ДоКАЗАТЕЛЬСТво. В предположении противного для любого натурального числа $n$ найдется такой оператор $\mathscr{A}_{n}$ из $\mathfrak{M}_{p}(E)$, что $\theta\left(\mathscr{A}_{n}, \mathscr{A}_{0}\right)<1 / n$, и в то же время сушествует ненулевое решение $u_{n}$ включения $0 \in u^{\prime}+\mathscr{A}_{n}(u)$, $\left\|u_{n} ; C_{b}(R, E)\right\|=R_{n}<\infty$. Не ограничивая обшности (см. доказательство леммы 5), можно считать, что $R_{n}=1,\left|u_{n}(0)\right|>1 / 2$ и последовательность $u_{n}$ сходится к некоторой функции $u: \mathbb{R} \rightarrow E$ равномерно на каждом ограниченном подмножестве $\mathbb{R}$. Предельная функция $u$ ограничена: $|u(t)| \leqslant 1$, отлична от нуля: $|u(0)| \geqslant 1 / 2$, и согласно лемме 1 является решением включения $0 \in u^{\prime}+\mathscr{A}_{0}(u)$. Но это противоречит условию $\mathscr{A}_{0} \in \mathfrak{N}_{p}(E)$. Лемма доказана.

Будем говорить, что семейство операторов $\mathscr{A}_{\lambda}, 0 \leqslant \lambda \leqslant 1$, соединяет операторы $\mathscr{A}_{0}, \mathscr{A}_{1}$ класса $\mathfrak{N}_{p}(E)$, если $\mathscr{A}_{\lambda} \in \mathfrak{N}_{p}(E) \forall \lambda \in[0,1]$ и $\theta\left(\mathscr{A}_{\mu}, \mathscr{A}_{\lambda}\right) \rightarrow 0$ при $\mu \rightarrow \lambda$; операторы $\mathscr{A}_{0}, \mathscr{A}_{1}$ назовем гомотопнылми в классе $\mathfrak{N}_{p}(E)$. Отношение гомотопности есть эквивалентность. Соответствуюшие классы эквивалентности назовем компонентами множества $\mathfrak{N}_{p}(E)$. Из леммы 6 следует, что в каждой компоненте $\mathfrak{N}_{p}(E)$ имеется однозначный оператор.

Обозначим через $\mathfrak{K}_{p}(E)$ совокупность таких операторов $\mathscr{A}$ класса $\mathfrak{N}_{p}(E)$, что для любого многозначного отображения $\mathscr{F}: \mathbb{R} \times E \rightarrow E$, удовлетворяющего условиям I), II) и равенству (18), включение (9) имеет ограниченное решение.

ЛЕмма 7. Однозначный оператор $\mathscr{A}$ класса $\mathfrak{N}_{p}(E)$ принадлежит классу $\mathfrak{K}_{p}(E)$ в том и только том случае, если он обладает свойством 
$(k)_{p}$ для любого непрерывного отображения $f: \mathbb{R} \times E \rightarrow E$, удовлетворяющего условию

$$
\lim _{R \rightarrow \infty} \frac{1}{R^{p-1}} \sup _{|v| \leqslant R, t}|f(t, v)-\mathscr{A}(v)|=0
$$

дифференциальное уравнение $0=u^{\prime}+f(t, u)$ имеет ограниченное решение.

ДОКАЗАТЕЛЬСТвО. Необходимость свойства $(k)_{p}$ для справедливости включения $\mathscr{A} \in \mathfrak{K}_{p}(E)$ очевидна. Остановимся на доказательстве достаточности. Пусть оператор $\mathscr{A}$ обладает свойством $(k)_{p} ; \mathscr{F}: \mathbb{R} \times E \rightarrow \mathrm{Cv} E$ - многозначное отображение, удовлетворяющее условиям I), II) и равенству (18). Для каждого $\varepsilon>0$ существует однозначная и непрерывная $\varepsilon$-аппроксимация $f_{\varepsilon}: \mathbb{R} \times E \rightarrow E$ отображения $\mathscr{F}: \mathbb{R} \times E \rightarrow \mathrm{Cv} E, \theta_{E^{2}}\left(f_{\varepsilon}, \mathrm{Gr} \mathscr{F}\right)<\varepsilon[4]$. Отсюда легко выводится, что при любом $\varepsilon>0$ отображение $f=f_{\varepsilon}$ удовлетворяет условию (27). Поэтому существуют ограниченные решения $x_{\varepsilon}$ дифференциальных уравнений $0=x^{\prime}+f_{\varepsilon}(t, x)$. Используя включение $\mathscr{A} \in \mathfrak{N}_{p}(E)$, можно по обычной схеме [5, с. 331-337] установить оценку $\left|x_{\varepsilon}(t)\right|<R_{0}$, константа $R_{0}$ не зависит от $t$ и $\varepsilon$. Семейство функций $x_{\varepsilon}(t), 0<\varepsilon<1$, равномерно ограничено и равностепенно непрерывно на прямой $\mathbb{R}$. Если $x(t)$ - предельная для семейства $x_{\varepsilon}(t)$ функция (в топологии равномерной сходимости на компактах), то $|x(t)| \leqslant R_{0}$ и $x$ есть решение включения (9). Лемма доказана.

Свойство $(k)_{p}$ было введено и изучено в статье [8]; там же указаны широкие достаточные условия для включения $\mathscr{A} \in \mathfrak{K}_{p}(E), \mathscr{A}$ - однозначный оператор класса $\mathfrak{N}_{p}(E)$. Ниже результаты [8] переносятся на многозначные операторы.

Лемма 8. Eсли $A_{0} \in \mathfrak{K}_{p}(E), \operatorname{co}\left(\mathscr{A}_{0}, \mathscr{A}_{1}\right) \in \mathfrak{N}_{p}(E)$, mo $\mathscr{A}_{1} \in \mathfrak{K}_{p}(E)$.

ДоказАтельСтво. Пусть $\mathscr{A}=\operatorname{co}\left(\mathscr{A}_{0}, \mathscr{A}_{1}\right)$, многозначное отображение $\mathscr{F}$ : $\mathbb{R} \times E \rightarrow \mathrm{Cv} E$ удовлетворяет условиям I), II) и равномерно асимптотически близко к оператору $\mathscr{A}_{1}$. Многозначное отображение $\mathscr{F}_{1}(t, v)=\operatorname{co}\left(\mathscr{F}(t, v), \mathscr{A}_{0}(v)\right)$ равномерно асимптотически близко к оператору $\mathscr{A}$ класса $\mathfrak{N}_{p}(E)$, поэтому совокупность ограниченных решений $x$ дифференциального включения $0 \in x^{\prime}+\mathscr{F}_{1}(t, x)$ есть ограниченное в $C_{b}(\mathbb{R}, E)$ множество: $\left\|x ; C_{b}(\mathbb{R}, E)\right\|<R_{0}$. В частности, подобная оценка верна для ограниченных решений дифференциальных включений вида

$$
0 \in u^{\prime}+\varphi(|u|) \mathscr{F}(t, u)+(1-\varphi(|u|)) \mathscr{A}_{0}(u),
$$

где $\varphi:[0, \infty) \rightarrow[0,1]$ - финитные непрерывные функции.

Пусть $\varphi(s)=1$ при $0 \leqslant s \leqslant R_{0}, 0<\varphi(s)<1$ при $R_{0}<s<R_{0}+1$ и $\varphi(s)=0$ при $s>R_{0}+1$. Отображение $\varphi(|v|) \mathscr{F}(t, v)+(1-\varphi(|v|)) \mathscr{A}_{0}(v)$ равномерно асимптотически близко к оператору $\mathscr{A}_{0}$ класса $\mathfrak{K}_{p}(E)$, поэтому включение $(28)$ имеет решение $x$, для которого $|x(t)| \leqslant R_{0}$. Следовательно, $1=\varphi(|x(t)|)$, поэтому $x$ есть решения включения (9). Лемма доказана.

Ее усилением является

Teорема 4. Eсли операторы $\mathscr{A}_{0}, \mathscr{A}_{1}$ гомотопны в классе $\mathfrak{N}_{p}(E)$ и $\mathscr{A}_{0} \in$ $\mathfrak{K}_{p}(E)$, mo $\mathscr{A}_{1} \in \mathfrak{K}_{p}(E)$. 
ДокаЗАТЕЛЬСТво. Пусть $\mathscr{A}_{\lambda}, 0 \leqslant \lambda \leqslant 1,-$ семейство операторов класса $\mathfrak{N}_{p}(E)$, соединяющее $\mathscr{A}_{0}, \mathscr{A}_{1}$. Множество $\Lambda=\left\{\lambda \in[0,1], \mathscr{A}_{\lambda} \in \mathfrak{K}_{p}(E)\right\}$ содержит нуль, поэтому непусто. Если $\lambda \in \Lambda, \mu \in[0,1]$ и $|\lambda-\mu|<\delta$, то при достаточно малых $\delta$ в силу леммы $6 \operatorname{co}\left(\mathscr{A}_{\lambda}, \mathscr{A}_{\mu}\right) \in \mathfrak{N}_{p}(E)$. Согласно лемме $8 \mathscr{A}_{\mu} \in \mathfrak{K}_{p}(E)$, т.е. $\mu \in \Lambda$ и, таким образом, $\Lambda$ - открытое множество в $[0,1]$. Если $\lambda_{i} \in \Lambda$ и $\lambda_{i} \rightarrow \lambda$, то $\operatorname{co}\left(\mathscr{A}_{\lambda_{i}}, \mathscr{A}_{\lambda}\right) \in \mathfrak{N}_{p}(E)$ при больших $i$, следовательно, $\lambda \in \Lambda$. Итак, $\Lambda=[0,1]$, в частности, $\mathscr{A}_{1} \in \mathfrak{K}_{p}(E)$. Теорема доказана.

Теорема 4 означает, что каждая компонента $\mathfrak{N}_{p}(E)$ либо вся входит в класс $\mathfrak{K}_{p}(E)$, либо не пересекается с этим классом. В первом случае соответствуюшую компоненту назовем, следуя [8], компонентой существования. Очевидно, в каждой компоненте сушествования найдется однозначньй оператор класса $\mathfrak{K}_{p}(E)$.

Лемма 9. Пусть $\mathscr{A} \in \mathfrak{N}_{p}(E) u \operatorname{ind}(0, \mathscr{A}) \neq 0$. Тогда $\mathscr{A} \in \mathfrak{K}_{p}(E)$.

ДокАЗАТЕЛЬСТво. Пусть $\mathscr{A}_{0}$ - однозначный оператор класса $\mathfrak{N}_{p}(E)$, гомотопньй оператору $\mathscr{A}$. Тогда ind $\left(0, \mathscr{A}_{0}\right)=\operatorname{ind}(0, \mathscr{A}) \neq 0$. В силу известных результатов $[5, \S 14],[8] \mathscr{A}_{0}$ обладает свойством $(k)_{p}$, поэтому согласно теореме 4 и лемме 7 $\mathscr{A} \in \mathfrak{K}_{p}(E)$. Лемма доказана.

ПРЕДЛОЖЕнИЕ 4 [8]. Пусть $\Phi: E \rightarrow \mathbb{R}$ - гладкая 2-однородная невырожденная функиия и множество $\mathfrak{R}=\{v \in E,|v|=1, \Phi(v)<0\}$ нестягиваемо по себе в точку. Тогда $\nabla \Phi \in \mathfrak{K}_{2}(E)$.

6. Обобщим результаты предшествующего пункта на случай бесконечномерного пространства $V$. Пусть многозначное отображение $\mathscr{F}: \mathbb{R} \times V \rightarrow V^{*}$ удовлетворяет условиям I), II), порождаемый $\mathscr{F}$ оператор суперпозиции $F$ принадлежит классу $\alpha_{1}(Y(T), Z(T))$ для любого отрезка $T \subset \mathbb{R}$ и, наконец, выполнено условие (18) равномерной асимптотической однородности $\mathscr{F}$ с некоторым оператором $\mathscr{A}$ класса $\mathfrak{M}_{p}(V), 1<p<\infty$. При этих предположениях справедлива

Teорема 5. Пусть $\mathscr{A} \in \mathfrak{N}_{p}(V) u \operatorname{ind}(0, \mathscr{A}) \neq 0$. Тогда дифференииальное включение (9) имеет ограниченное решение.

ДокАЗАТЕЛЬСТво. Пусть $E_{n}$ - исчерпьвающая $V$ последовательность конечномерных подпространств, $j_{n}, j_{n}^{*}$ - порождаемые $E_{n}$ операторы. В силу леммы 5 и определения индекса найдется такой номер $n_{0}$, что $j_{n}^{*} \mathscr{A} j_{n} \in \mathfrak{N}_{p}\left(E_{n}\right)$ и $\operatorname{ind}\left(0, j_{n}^{*} \mathscr{A} j_{n}\right)=\operatorname{ind}(0, \mathscr{A}) \neq 0$ при $n>n_{0}$. Согласно лемме $9 j_{n}^{*} \mathscr{A} j_{n} \in \mathfrak{K}_{p}\left(E_{n}\right)$, поэтому включение вида (25) имеет ограниченное решение при $n>n_{0}$. Теперь существование ограниченного решения дифференциального включения (9) вытекает из леммы 4. Теорема доказана.

Остановимся на бесконечномерном варианте предложения 4. Напомним, что метрическое пространство $\mathfrak{R}$ называют гомологически тривиальнылм, если его группы гомологий изоморфны группам гомологий одноточечного пространства, и гомологически нетривиальным в противном случае.

Теорема 6. Пусть $\mathscr{A} \in \mathfrak{N}_{2}(V), \mathscr{A}$ - потенциальный оператор с гладким потенциалом $\Phi, \quad \Phi(0)=0$, и метрическое пространство $\mathfrak{R}=\{v \in V$, $\|v\|=1, \Phi(v)<0\}$ гомологически нетривиально. Тогда включение (9) имеет ограниченное решение. 
ДОКАЗАТЕЛЬСТВО. В силу теоремы 3 и леммы 5 найдется такой индекс $n_{0}$, что пространство $\Re \cap E_{n}$ гомологически нетривиально и $j_{n}^{*} \mathscr{A} j_{n} \in \mathfrak{N}_{p}\left(E_{n}\right)$ при $n>n_{0}$. В частности, пространство $\mathfrak{R} \cap E_{n}=\left\{v \in E_{n},\|v\|=1, \Phi(v)<0\right\}$ нестягиваемо, поэтому $j_{n}^{*} \mathscr{A}_{n} \in \mathfrak{K}_{p}\left(E_{n}\right)$. Следовательно, дифференциальное включение $(25)$ имеет ограниченное решение при $n>n_{0}$. Теперь требуемьй результат вытекает из леммы 4. Теорема доказана.

Полезно заметить, что в условиях теоремы 6 может выполняться равенство $\operatorname{ind}(0, \mathscr{A})=0$. Такого рода примеры для конечномерного пространства $V$ рассмотрены в [8]. Отметим определенную аналогию теорем 1 и 5 . В условиях теоремы 1 гарантируется сушествование стационарного решения автономного дифференциального включения $0 \in y^{\prime}+\mathscr{F}(y)$.

Для применения теорем 5, 6 желательно располагать примерами операторов класса $\mathfrak{N}_{p}(V)$. Сравнительно просто подобные примеры конструируются для потенциальных операторов.

ЛЕмма 10. Пусть $\Phi: V \rightarrow \mathbb{R}-$ гладкий р-однородный невырожденный функиионал и $\Phi^{\prime} \in \mathfrak{M}_{p}(V), \quad p \geqslant 2$. Тогда $\Phi^{\prime} \in \mathfrak{N}_{p}(V)$.

ДокАЗАТЕЛЬСТво. Пусть $y_{0}$ - ограниченное решение дифференциального уравнения $0=y^{\prime}+\Phi^{\prime}(y)$. Докажем равенство $y_{0}(t)=0$. Вначале установим, что при любом $\alpha$ из $\mathbb{R} y_{0}(t)$ - единственное решение задачи Коши $0=y^{\prime}+\Phi^{\prime}(y)$, $y(\alpha)=y_{0}(\alpha)$. Действительно, если $y_{1}(t)$ - еше одно решение этой же задачи, то разность $u(t)=y_{1}(t)-y_{0}(t)$ удовлетворяет соотношениям $u^{\prime}(t)=\Phi^{\prime}\left(y_{0}\right)-\Phi^{\prime}\left(y_{1}\right)$, $u(\alpha)=0$. Используя равенство $\left(|u(t)|^{2}\right)^{\prime}=2\left(u(t), u^{\prime}(t)\right)$, получаем

$$
\begin{aligned}
\frac{|u(t)|^{2}}{2}= & \int_{\alpha}^{t}\left(y_{1}(s)-y_{0}(s), \Phi^{\prime}\left(y_{0}(s)\right)-\Phi^{\prime}\left(y_{1}(s)\right)\right) d s \leqslant k_{0} \int_{\alpha}^{t}|u(s)|^{p} d s \\
& -k_{1} \int_{\alpha}^{t}\|u(s)\|^{p} d s \leqslant k_{0} \int_{\alpha}^{t}|u(s)|^{p} d s, \quad t>\alpha,
\end{aligned}
$$

откуда вытекает равенство $u(t)=0$, т.е. $y_{1}(t)=y_{0}(t)$.

Фиксируем $\alpha \in \mathbb{R}$ так, что $y_{0}(\alpha) \in V$. Пусть $E_{n}, j_{n}, j_{n}^{*}$ - пространства и операторы, фигурируюшие в методе Галёркина. Подберем последовательность $h_{n}$ из $E_{n}$, сходящуюся к $y_{0}(\alpha)$ в метрике $V$.

Рассмотрим задачу Коши

$$
0=y^{\prime}+j_{n}^{*} \Phi^{\prime}\left(j_{n} y\right), \quad y(\alpha)=h_{n} .
$$

Для каждого отрезка $T=[\alpha, \omega]$ найдется номер $N=N(T)$ такой, что при $n>$ $N(T)$ задача Коши (29) имеет решение $y_{n}(t), \alpha \leqslant t \leqslant \omega$; если $z_{n}(t)=\Phi^{\prime}\left(y_{n}(t)\right)$, $z_{0}(t)=\Phi^{\prime}\left(y_{0}(t)\right)$, то в силу леммы 2 и результатов работы [17]

$$
\begin{gathered}
y_{n} \rightarrow y_{0} \quad \text { в } \quad L^{p}(T, V) \cap C(T, H), \quad z_{n} \rightarrow z_{0} \quad \text { в } L^{q}\left(T, V^{*}\right), \\
y_{n}(t) \rightarrow y_{0}(t) \quad \text { в } V \text { п.в. на } T, \quad z_{n}(t) \rightarrow z_{0}(t) \text { в } V^{*} \text { п.в. на } T .
\end{gathered}
$$

Так как $y_{n}^{\prime}=-j_{n}^{*} z_{n}$, то $\left(\Phi\left(y_{n}\right)\right)^{\prime}=-\left|j_{n}^{*} z_{n}\right|^{2}$ и

$$
\Phi\left(y_{n}(t)\right)-\Phi\left(y_{n}(\alpha)\right)=-\int_{\alpha}^{t}\left|j_{n}^{*} z_{n}(s)\right|^{2} d s .
$$


С другой стороны,

$$
p \Phi\left(y_{n}\right)=\left(y_{n}, \Phi^{\prime}\left(y_{n}\right)\right)=\left(y_{n}, z_{n}\right) \geqslant k_{1}\left\|y_{n}\right\|^{p}-k_{0}\left|y_{n}\right|^{p},
$$

следовательно,

$$
k_{1}\left\|y_{n}(t)\right\|^{p} \leqslant k_{0}\left|y_{n}(t)\right|^{p}+p \Phi\left(y_{n}(t)\right) \leqslant k_{0}\left|y_{n}(t)\right|^{p}+p \Phi\left(y_{n}(\alpha)\right) .
$$

Поэтому последовательность $\left\|y_{n}(t)\right\|$ ограничена: $\left\|y_{n}(t)\right\| \leqslant R_{1}$; последовательность $z_{n}(t)$ ограничена в пространстве $V^{*}:\left\|z_{n}(t)\right\|_{*} \leqslant R_{2}$. В дополнение к (30) можно считать, что $y_{n} \rightarrow y$ в $C_{w}(T, V)$. Проведенные рассуждения показывают, в частности, что в качестве $\alpha$ можно взять любое число из $\mathbb{R}$.

Имеем далее

$$
\left|p \Phi\left(y_{n}\right)\right|=\left|\left(y_{n}, z_{n}\right)\right| \leqslant\left|j_{n} y_{n}\right|\left|j_{n}^{*} z_{n}\right| \leqslant c_{n}\left|j_{n}^{*} z_{n}\right|,
$$

где $c_{n}=\left\|y_{n} ; C(T, H)\right\|+1$. Неравенство (31) и последняя оценка приводят к соотношению

$$
\Phi\left(y_{n}(t)\right)-\Phi\left(y_{n}(\alpha)\right) \leqslant-\int_{\alpha}^{t} \frac{p^{2}}{c_{n}^{2}} \Phi^{2}\left(y_{n}(s)\right) d s .
$$

Поскольку $y_{n}(t) \rightarrow y_{0}(t)$ в $\sigma\left(V, V^{*}\right), y_{n}(\alpha) \rightarrow y(\alpha)$ в $V$, а последовательность $\Phi\left(y_{n}(s)\right)$ сходится почти всюду к $\Phi\left(y_{0}(s)\right)$ и ограничена, то в пределе получаем оценку

$$
\Phi\left(y_{0}(t)\right)-\Phi\left(y_{0}(\alpha)\right) \leqslant-\int_{\alpha}^{t} \frac{p^{2}}{c^{2}} \Phi^{2}\left(y_{0}(s)\right) d s,
$$

в которой $c=\left\|y_{0} ; C(T, H)\right\|+1$. Оценка (32) верна для любых $t>\alpha$. Из нее без труда вытекает равенство $\Phi\left(y_{0}(t)\right)=0$. Но тогда

$$
0=\Phi\left(y_{0}(\omega)\right)-\Phi\left(y_{0}(\alpha)\right)=-\lim _{n \rightarrow \infty} \int_{\alpha}^{\omega}\left|j_{n}^{*} z_{n}(s)\right|^{2} d s
$$

в частности, $j_{n}^{*} z_{n}(s) \rightarrow 0$ в $H$ почти при всех $s$. Если $j_{n}^{*} z_{n}\left(s_{0}\right) \rightarrow 0$ в $H$, то для любого $v$ из $E_{k}$ при $n>k$ имеем $\left(v, z_{n}\left(s_{0}\right)\right)=\left(v, j_{n}^{*} z_{n}\left(s_{0}\right)\right) \rightarrow 0$ при $n \rightarrow \infty$. Так как объединение пространств $E_{k}, k=1,2, \ldots$, всюду плотно в пространстве $V$, то $z_{n}\left(s_{0}\right) \rightarrow 0$ в $\sigma\left(V^{*}, V\right)$. Из проведенных рассуждений следует равенство $z_{0}(t)=$ $\Phi^{\prime}\left(y_{0}(t)\right)=0$ п.в. Поскольку $\Phi-$ невырожденный функционал, то $y_{0}(t) \equiv 0$. Лемма доказана.

В качестве иллюстративного примера рассмотрим параболическое уравнение с одной пространственной переменной

$$
\frac{\partial y}{\partial t}-\frac{\partial}{\partial x}\left(a\left|\frac{\partial y}{\partial x}\right|^{p-2} \frac{\partial y}{\partial x}\right)+(b-\lambda c)|y|^{p-2} y+g\left(t, x, y, \frac{\partial y}{\partial x}\right)=0,
$$

где $x \in \Omega=(0,1), t \in \mathbb{R}, a \in C^{1}(\bar{\Omega}), a(x)>0, b \in C(\bar{\Omega}), b(x) \geqslant 0, c \in C(\bar{\Omega})$, $c(x)>0, \lambda \in \mathbb{R}, g(t, x, \eta, \xi)$ - функция, определенная и непрерывная на $\mathbb{R} \times \bar{\Omega} \times \mathbb{R}^{2}$,

$$
|g(t, x, \eta, \xi)| \leqslant \operatorname{const}\left(1+|\eta|^{r}+|\xi|^{r}\right),
$$


$2 \leqslant p<\infty, 1<r<p-1$. Применим предшествуюшие результаты, считая, что $V$ есть пространство Соболева $\stackrel{\circ}{L}_{p}^{1}(\Omega)$ функций $v: \Omega \rightarrow \mathbb{R}$, удовлетворяющих нулевому граничному условию: $\left.v\right|_{\partial \Omega}=0, H=L^{2}(\Omega)$. Пара пространств $V, H$ однозначно определяет соответствуюшие им пространства $\mathrm{BS}^{p}(\mathbb{R}, V), C_{b}(\mathbb{R}, H)$, которые могут быть интерпретированы как пространства функций на полосе $\Omega \times \mathbb{R}$; функции $y$ из $\mathrm{BS}^{p}(\mathbb{R}, V)$ удовлетворяют нулевому граничному условию

$$
y(0, t)=y(1, t)=0, \quad t \in \mathbb{R} .
$$

Под ограниченным решением задачи (33), (35) понимается ограниченное решение включения (9), где однозначное отображение $\mathscr{F}: \mathbb{R} \times V \rightarrow V^{*}$ определяется равенством

$$
(w, \mathscr{F}(t, v))=\int_{\Omega}\left(w^{\prime} a\left|v^{\prime}\right|^{p-2} v^{\prime}+w(b-\lambda c)|v|^{p-2} v+w g\left(t, x, v, v^{\prime}\right)\right) d x \quad \forall w \in V .
$$

В силу результатов работы [18] порождаемый $\mathscr{F}$ оператор суперпозиции $F$ принадлежит классу $\alpha_{1}(Y(T), Z(T))$ для любого отрезка $T \subset \mathbb{R}$. Из оценки (34) вытекает, что отображение $\mathscr{F}$ равномерно асимптотически однородно, его главная часть $\mathscr{A}: V \rightarrow V^{*}$ определена равенством

$$
(w, \mathscr{A}(v))=\int_{\Omega}\left(w a\left|v^{\prime}\right|^{p-2} v^{\prime}+w(b-\lambda c)|v|^{p-2} v\right) d x \quad \forall w \in V .
$$

Оператор $\mathscr{A}$ потенциален. Ненулевые особые точки $\mathscr{A}$ являются ненулевыми решениями нелинейной краевой задачи

$$
-\left(a\left|u^{\prime}\right|^{p-2} u^{\prime}\right)^{\prime}+(b-\lambda c)|u|^{p-2} u=0, \quad u(0)=u(1)=0 ;
$$

числа $\lambda$ называют собственными значениями задачи (36). Наименьшее собственное значение $\lambda_{1}>0$. Если $\lambda_{1} c(x)-b(x)>0$, то у задачи (36) точно счетное множество собственных значений $0<\lambda_{1}<\lambda_{2}<\cdots$, причем $\lambda_{n} \rightarrow \infty$ [22].

При сформулированных выше предположениях относительно функций $a, b, c, g$ справедлива

ТЕОРема 7. Если $\lambda \neq \lambda_{n}, \quad n=1,2, \ldots$, то задача (33), (35) имеет ограниченное решение.

ДокАЗАТЕЛЬство. Действительно, если $\lambda \neq \lambda_{n}, n=1,2, \ldots$, то $\mathscr{A}$ - регулярньй на $\infty$ потенциальньй оператор. Согласно лемме $10 \mathscr{A} \in \mathfrak{N}_{p}(V)$, а так как $\mathscr{A}-$ нечетный оператор, то ind $(0, \mathscr{A}) \neq 0$. Теперь требуемый результат вытекает из теоремы 5. Теорема доказана.

При $\lambda \gg 1$ оператор $\mathscr{A}$ немонотонен. Следовательно, в отличие от [23], [24] наши результаты применимы к параболическим уравнениям с немонотонной главной частью. Аналогичные теореме 7 утверждения имеют место для широкого класса параболических уравнений (здесь оказываются полезными установленные в [25] обобшения результатов статьи [22]).

Развитая выше схема применима и к задаче о периодических решениях дифференциального включения (9). Не останавливаясь на формулировках бесконечномерных многозначных аналогов известных результатов (см., например, [5, c. 331-338]), отметим, что если в условиях теоремы 7 функция $g$ периодична по $t$, то задача (33), (35) имеет ограниченное решение того же периода. 


\section{Список литературы}

1. Похожаев С.И. О разрешимости нелинейных уравнений с нечетными операторами // Функцион. анализ и его прилож. 1967. Т. 1. № 3. С. 66-73.

2. Скрыпник И. В. Методы исследования нелинейных эллиптических граничных задач. М.: Наука, 1990.

3. Browder F. E. Nonlinear elliptic boundary value problems and the generalized topological degree // Bull. Amer. Math. Soc. 1970. V. 76. № 5. P. 999-1005.

4. Борисович Ю. Г., Гельман Б. Д., Мышкис А. Д., Обуховский В. В. Топологические методы в теории неподвижных точек многозначных отображений // УМН. 1980. Т. 35. №1. C. 59-126.

5. Красносельский М. А., Забрейко П. П. Геометрические методы нелинейного анализа. М.: Наука, 1975.

6. Скрыпник И. В. Нелинейные эллиптические уравнения высшего порядка. Киев: Наук. думка, 1973.

7. Кларк Ф. Оптимизация и негладкий анализ. М.: Наука, 1988.

8. Мухамадиев Э. Ограниченные решения и гомотопические инварианты систем нелинейных дифференциальных уравнений // Докл. РАН. 1996. Т. 351. № 5. С. 596-598.

9. Климов B.C. О топологических характеристиках негладких функционалов // Изв. РАН. Сер. матем. 1998. Т. 62. № 5. С. 117-134.

10. Масси У. Теория гомологий и когомологий. М.: Мир, 1981.

11. Стинрод Н., Эйленберг C. Основания алгебраической топологии. М.: ИЛ, 1958.

12. Гаевский $Г$., Грёзер K., Захариас $K$. Нелинейные операторные уравнения и операторные дифференциальные уравнения. М.: Мир, 1978.

13. Климов B.C. К задаче о периодических решениях операторных дифференциальных включений // Изв. АН СССР. Сер. матем. 1989. Т. 53. № 2. С. 309-327.

14. Климов В.С., Сенчакова Н. В. Об относительном вращении многозначных векторных полей // Матем. сб. 1991. Т. 182. № 10. С. 1393-1407.

15. Обен Ж. П., Экланд И. Прикладной нелинейный анализ. М.: Мир, 1988.

16. Скрыпник И. В. Разрешимость и свойства решений нелинейных эллиптических уравнений // Современные проблемы математики. 1976. Т. 9. С. 131-254.

17. Климов B. C. Эволюционные параболические неравенства с многозначными операторами // Матем. сб. 1993. Т. 184. № 8. С. 37-54.

18. Климов В.C. Об операторе сдвига по траекториям параболических включений // Дифф. уравн. 1995. Т. 31. №10. С. 1719-1724.

19. Дубинский Ю. А. Нелинейные эллиптические и параболические уравнения // Современные проблемы математики. 1976. Т. 9. С. 5-130.

20. Красовский Н. Н. Некоторые задачи теории устойчивости движения. М.: Физматгиз, 1959.

21. Castaing C., Valadier M. Convex analysis and measurable multifunctions // Lecture Notes in Math. № 580. Berlin: Springer, 1977.

22. Нечас И. О дискретности спектра нелинейного уравнения Штурма-Лиувилля // ДАН CCCP. 1971. T. 201. № 5. C. 1045-1048.

23. Левитан Б.М., Жиков В.В. Почти периодические функции и дифференциальные уравнения. М.: Изд-во МГУ, 1978.

24. Панков А. А. Ограниченные и почти периодические решения нелинейных дифференциально-операторных уравнений. Киев: Наук. думка, 1985.

25. Fučik S., Nečas J., Souček J., Souček V. Spectral analysis of nonlinear operators // Lecture Notes in Mathematics. № 346. Berlin: Springer, 1973. 